\title{
Optimizing Expected Utility of Dividend Payments for a Brownian Risk Process and a Peculiar Nonlinear ODE
}

\author{
Friedrich Hubalek* ${ }^{*}$, Walter Schachermayer \\ Department of Financial and Actuarial Mathematics, Vienna University of \\ Technology, Wiedner Hauptstraße 8-10/105, A-1040 Vienna, Austria
}

\begin{abstract}
We consider the problem of maximizing the expected utility of discounted dividend payments of an insurance company. The risk process, describing the insurance business of the company, is modeled as Brownian motion with drift. We mainly consider power utility and special emphasis is given to the limiting behavior when the coefficient of risk aversion tends to zero. We then find convergence of the corresponding dividend strategies to the classical case of maximizing the expected dividend payments.
\end{abstract}

Key words: Optimal dividends, risk process, optimal control, nonlinear ODE

\section{Introduction}

The question of optimizing the dividend policy of an insurance company is a classical problem of actuarial mathematics. The typical way to mathematically formalize this problem (see Gerber, 1979, Ch. 10.1) is to model the reserve process $\left(R_{t}\right)_{t \geq 0}$ of an insurance company (before dividend payments) in the classical Cramèr-Lundberg way using a compound Poisson process. A dividend paying strategy then consists in an adapted increasing process $\left(C_{t}\right)_{t \geq 0}$, modeling the accumulated dividend payments up to time $t$. Define the ex-dividend process $X$ by letting

$$
X_{t}=R_{t}-C_{t}, \quad(t \geq 0),
$$

\footnotetext{
* Corresponding author.

Email address: fhubalek@fam.tuwien.ac.at (Friedrich Hubalek).
} 
and the time of ruin under the dividend paying policy $C$ by

$$
\tau=\inf \left\{t: X_{t} \leq 0\right\}
$$

Both $X$ and $\tau$ depend obviously on $C$. A dividend policy $C$ is admissible if $C_{t}=C_{\tau-}$ for $t \geq \tau$ : after (as well as at) the moment of ruin, no dividend payments are possible any more.

To find the optimal dividend policy one has to define the target functional which has to be maximized. The classical choice is the expected value of discounted dividend payments, i.e.,

$$
\mathbb{E}\left[\int_{0}^{\infty} e^{-\beta t} d C_{t}\right] \mapsto \max !,
$$

where $\beta>0$ is a fixed discount rate. In this case one finds (under suitable regularity conditions, see Gerber (1979)) that the optimal strategy is of barrier type, i.e., there is a number $B>0$ such that the company pays dividends whenever the ex-dividend reserve process $X$ hits the level $B$. In the setting where $R$ is modeled as Brownian motion with drift an analogous result was obtained by Asmussen and Taksar (1997), and we shall review this result below.

In the present paper we study a variation of the optimization problem (3): instead of maximizing the expected value of discounted dividend payments, we maximize the expected value of the utility of these payments, for some utility function $U: \mathbb{R}_{\geq 0} \rightarrow \mathbb{R}_{\geq 0}$. To make this idea meaningful we require apart from the usual assumptions of monotonicity, smoothness and concavity of $U$ - that $U(0)=0$, as well as the Inada conditions $U^{\prime}(0)=\lim _{x \backslash 0} U^{\prime}(x)=\infty$ and $U^{\prime}(\infty)=\lim _{x \nearrow \infty} U^{\prime}(x)=0$. In fact, we shall focus our attention on the case of power utility

$$
U(x)=\frac{x^{\alpha}}{\alpha}, \quad(x \geq 0),
$$

where we let $\alpha$ vary in $(0,1)$. Of course, the limiting case $\alpha=1$ corresponds to the classical problem (3). We shall be particularly interested in the limiting behavior as $\alpha$ tends to 1 .

If $\alpha<1$ we may suppose without loss of generality that $C$ is absolutely continuous with respect to Lebesgue measure, as the condition $U^{\prime}(\infty)=0$ (which is equivalent to $\lim _{x \rightarrow \infty} \frac{U(x)}{x}=0$ for concave functions), implies that the singular part of $C$ does not contribute to the target functional. Hence we suppose that 
the process $\left(C_{t}\right)_{t \geq 0}$ admits almost surely a density process, denoted by $\left(c_{t}\right)_{t \geq 0}$ modeling the intensity of the dividend payments in continuous time. We then have for each $t \geq 0$

$$
C_{t}=\int_{0}^{t} c_{s} d s, \quad \text { a.s. }
$$

Now we can define, for a fixed utility function $U$, the target functional

$$
\mathbb{E}\left[\int_{0}^{\infty} e^{-\beta t} U\left(c_{t}\right) d t\right] \mapsto \max !
$$

To make this problem - which will turn out to be quite delicate - more tractable we restrict ourself to the diffusion limit of the Cramèr-Lundberg model: we suppose that $R$ is simply Brownian motion with drift, i.e.,

$$
R_{t}=x+\mu t+\sigma W_{t}, \quad(t \geq 0)
$$

where $x>0$ denotes the initial reserve, $\mu \in \mathbb{R}$ the drift parameter, $\sigma>0$ the diffusion parameter and $W$ is a standard Brownian motion, defined on (and adapted to) some filtered probability space $(\Omega, \mathcal{F}, P, \mathbb{F})$ with $\mathbb{F}=\left(\mathcal{F}_{t}\right)_{t \geq 0}$. The case of a process $R$ of Cramèr-Lundberg type with jumps (of which the above process is a limiting case) is left to future research.

The ex-dividend process is now

$$
X_{t}=R_{t}-\int_{0}^{t} c_{s} d s, \quad(t \geq 0) .
$$

Recall that the dividend density process $\left(c_{t}\right)_{t \geq 0}$ is admissible, if it is a nonnegative adapted process, that satisfies $c_{t}=0$, for $t \geq \tau$, where $\tau$ is the time of ruin. As $X$ now is a continuous process we have $\tau=\inf \left\{t \geq 0: X_{t}=0\right\}$.

A word on the notation: as the process $\left(c_{t}\right)_{t \geq 0}$ is our control variable in the problem (3), it is customary in the control theory literature to denote this variable by $\left(u_{t}\right)_{t \geq 0}$. We prefer not to do so, as this might create confusion with the letter $U$, which denotes the utility function; we rather prefer to follow Björk et al. (1987) where a similar problem was investigated and where $\left(c_{t}\right)_{t \geq 0}$ had the interpretation of "consumption". 
According to the above discussion of admissible dividend densities we have

$$
\mathbb{E}\left[\int_{0}^{\infty} e^{-\beta t} U\left(c_{t}\right) d t\right]=\mathbb{E}\left[\int_{0}^{\tau} e^{-\beta t} U\left(c_{t}\right) d t\right] .
$$

We define the value function

$$
w(t, x)=\sup \mathbb{E}\left[\int_{t}^{\infty} e^{-\beta s} U\left(c_{s}\right) d s\right],
$$

where we maximize over all densities $\left(c_{s}\right)_{s \geq t}$ of admissible dividend paying processes starting at time $t$ conditionally on the assumption $X_{t}=R_{t}=x$. We may associate - at least formally - to the maximization problem (6) the Hamilton-Jacobi-Bellman (HJB) equation

$$
w_{t}+\sup _{c \geq 0}\left[(\mu-c) w_{x}+\frac{\sigma^{2}}{2} w_{x x}+U(c) e^{-\beta t}\right]=0, \quad w(t, 0)=0 .
$$

This is a nonlinear partial differential equation. The value function $w$ clearly is of the form

$$
w(t, x)=e^{-\beta t} v(x),
$$

where $v$ denotes a function depending only on the current reserve $x$. We have $w_{t}(t, x)=-\beta e^{-\beta t} v(x)$, hence we can simplify equation (11) to

$$
-\beta v+\sup _{c \geq 0}\left[(\mu-c) v_{x}+\frac{\sigma^{2}}{2} v_{x x}+U(c)\right]=0, \quad v(0)=0,
$$

which is a nonlinear ordinary differential equation. The supremum in (13) is attained at

$$
c=\left(U^{\prime}\right)^{-1}\left(v_{x}\right)=v_{x}^{-1 /(1-\alpha)}
$$

and (13) can be rewritten as

$$
\beta v-\mu v_{x}-\frac{\sigma^{2}}{2} v_{x x}-\frac{1-\alpha}{\alpha} v_{x}^{-\frac{\alpha}{1-\alpha}}=0, \quad v(0)=0 .
$$

We consider this ODE for $x \geq 0$. Apart from the obvious initial value $v(0)=0$ one more initial condition is clearly required to obtain a unique solution. We postpone this issue for the moment. 
There seems to be no closed form solution in terms of elementary functions (as we shall see below, there are good reasons why we should not expect an elementary solution).

We now summarize the main results of the paper.

Theorem 1 For $\alpha \in(0,1), \beta>0, \mu \in \mathbb{R}, \sigma>0$, the value function $w(t, x)$ defined in (10) equals $v(x) e^{-\beta t}$, where $v$ is the unique solution of the ODE (15), that has initial value $v(0)=0$, and is well-defined and concave on $\mathbb{R}_{\geq 0}$. The optimal dividend policy is given by $c_{t}=c\left(X_{t}\right)$, with

$$
c(x)=v_{x}(x)^{-\frac{1}{1-\alpha}} .
$$

The asymptotic behavior of $v(x), v_{x}(x)$, and $c(x)$, as $x \rightarrow \infty$, is given by

$$
\begin{aligned}
& v(x)=\left(\frac{1-\alpha}{\beta}\right)^{1-\alpha} \frac{x^{\alpha}}{\alpha}\left(1+\mathcal{O}\left(\frac{\ln x}{x}\right)\right), \quad x \rightarrow+\infty, \\
& v_{x}(x)=\left(\frac{1-\alpha}{\beta}\right)^{1-\alpha} x^{\alpha-1}\left(1+\mathcal{O}\left(\frac{\ln x}{x}\right)\right) \quad x \rightarrow+\infty, \\
& c(x)=\frac{\beta}{1-\alpha} x\left(1+\mathcal{O}\left(\frac{\ln x}{x}\right)\right), \quad x \rightarrow+\infty .
\end{aligned}
$$

To motivate the above asymptotic results we consider for a moment the situation where $\mu=0$ and $\sigma=0$, while $\alpha \in(0,1)$ and $\beta$ are still free. In other words, we consider the situation where the risk process $R$ is constant. In more pedestrian terms, we analyze the (rather trivial) question of consuming an initial amount $x$ of money by a (deterministic) consumption process $c$, such that $x=\int_{0}^{\infty} c_{t} d t$, which is optimal with respect to

$$
\int_{0}^{\infty} e^{-\beta s} U\left(c_{s}\right) d s \mapsto \max !
$$

An elementary calculation reveals that the leading term in (19) solves this problem, with the corresponding value function equal to the leading term in (17): the optimal consumption $c(x)$ is then proportional to the remaining wealth $x$, the proportionality factor being $\beta /(1-\alpha)$. Similarly the value function $v(x)$ then is proportional to the original utility function $U(x)=x^{\alpha} / \alpha$, the proportionality factor now being $((1-\alpha) / \beta)^{1-\alpha}$.

The above asymptotic formulas (17), (18), and (19), pertaining to the case of general $\mu \in \mathbb{R}$ and $\sigma>0$, may therefore very roughly be interpreted as 
follows: for very rich insurance companies, i.e., $x$ being close to infinity, the optimal dividend policy, as well as the corresponding value function, are almost the same as the optimal dividend policy for a company without any risky business, i.e., $\mu=0$ and $\sigma=0$. The explanation is that the influence of the risk process $R$, for fixed $\mu \in \mathbb{R}$ and $\sigma>0$, becomes irrelevant as compared to the present (huge) value $x$.

Let us now compare the theorem with the limiting case $\alpha=1$, i.e., the problem (3). In this case the HJB equation for the corresponding value function equals (by the same formal arguments as above)

$$
w_{t}+\sup _{c \geq 0}\left[(\mu-c) w_{x}+\frac{\sigma^{2}}{2} w_{x x}+c e^{-\beta t}\right]=0, \quad w(t, 0)=0,
$$

and the corresponding ODE reads

$$
-\beta v+\sup _{c \geq 0}\left[(\mu-c) v_{x}+\frac{\sigma^{2}}{2} v_{x x}+c\right]=0, \quad v(0)=0 .
$$

As long as $w_{x}(x, t)>e^{-\beta t}$ the optimal choice of $c$ is zero, which means that no dividend payments should take place. Note that the condition $w_{x}(x, t)>$ $e^{-\beta t}$ does not depend on $t$ and is equivalent to $v_{x}(x)>1$. For $v_{x}(x)=1$ the expression in the bracket does not depend on the choice of $c$, while for $v_{x}(x)<1$ the above equation becomes $w_{t}(t, x)=-\infty$ by letting $c$ tend to infinity, i.e., the equations (21) and (22) do not make sense any more.

Assuming $v_{x} \geq 1$ in (22) we obtain the ODE

$$
\beta v-\mu v_{x}-\frac{\sigma^{2}}{2} v_{x x}=0, \quad v(0)=0,
$$

which is very easy to solve. The basic solutions of (23) are $e^{\lambda_{1} x}$ and $e^{\lambda_{2} x}$, where

$$
\lambda_{1}=\frac{-\mu+\sqrt{\mu^{2}+2 \beta \sigma^{2}}}{\sigma^{2}}>0, \quad \lambda_{2}=\frac{-\mu-\sqrt{\mu^{2}+2 \beta \sigma^{2}}}{\sigma^{2}}<0 .
$$

As we must have $v(0)=0$ we get

$$
v(x)=k\left(e^{\lambda_{1} x}-e^{\lambda_{2} x}\right) .
$$

The parameter $k \in \mathbb{R}$ still is free to be chosen. As $v \geq 0$, we have $v_{x}(0)=$ $k\left(\lambda_{1}-\lambda_{2}\right) \geq 0$, whence $k>0$. The "good" choice of $k$ is an interesting issue and crucial for understanding the situation of Theorem 1 above. Note 
that $g(x)=e^{\lambda_{1} x}-e^{\lambda_{2} x}$ is a function on $\mathbb{R}_{\geq 0}$ which is concave on $\left[0, x_{0}\right]$, where $x_{0}=\frac{1}{\lambda_{1}-\lambda_{2}} \ln \left(\frac{\lambda_{2}^{2}}{\lambda_{1}^{2}}\right)$ is the unique inflection point of $g$; on $\left[x_{0}, \infty\right)$ the function $g$ is convex.
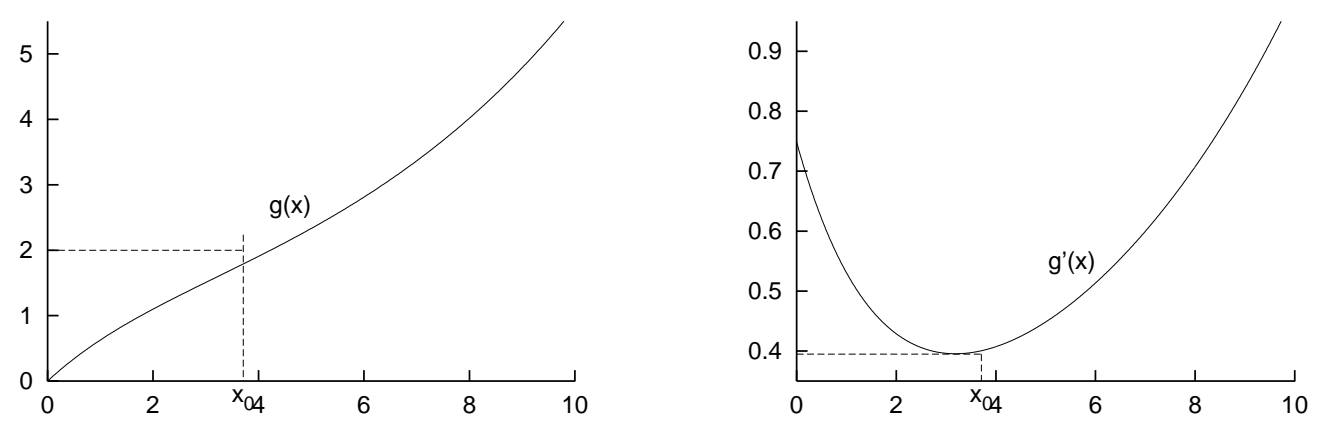

Fig. 1. The functions $g(x)$ and $g^{\prime}(x)$ with parameters $\beta=0.05, \mu=0.2, \sigma=1$

Hence there is a unique $k_{0}>0$, given by $k_{0}=\left(\lambda_{1} e^{\lambda_{1} x_{0}}-\lambda_{2} e^{\lambda_{2} x_{0}}\right)^{-1}$, such that, for $v(x)=k_{0} g(x)$, we have $v_{x}\left(x_{0}\right)=\min _{x \geq 0} v_{x}(x)=1$. It turns out that for this choice of $k$ the function $v$ is indeed the value function associated to the problem (3) on the interval $\left[0, x_{0}\right]$. At the inflection point $x_{0}$ is the optimal dividend barrier and beyond $x_{0}$, the value function $v$ is not given by the ODE (23) any more; it simply is continued in an affine way with slope 1 , i.e., $v(x)=v\left(x_{0}\right)+x-x_{0}$, for $x \geq x_{0}$. Clearly this continuation corresponds to the strategy of immediately paying all the reserve exceeding $x_{0}$ as dividends.

For the verification that this function $v$ is indeed the value function of the problem (3) we refer to the remarks at the end of Section 2 below. Note that, at the inflection point $x_{0}$ we have $v_{x x}=0$ and $v_{x}=1$, so that (22) yields $v\left(x_{0}\right)=\mu / \beta$ and that this quantity does not depend on $\sigma$.

It is interesting to discuss what happens for a choice of $k$ different from $k_{0}$. After all, $v(x)=k g(x)$ obeys the ODE (23) for all choices of $k$.

If $k<\left(\lambda_{1}-\lambda_{2}\right)^{-1}$, then the function $v=k g$ satisfies $v_{x}(0)<1$, hence the ODE (22) fails already to be well-defined at $x=0$. A more interesting choice is $\left(\lambda_{1}-\lambda_{2}\right)^{-1}<k<k_{0}$. In this case there is $0<x_{1}<x_{0}$ such that for $v=k g$ the ODE $(22)$ is satisfied on $\left[0, x_{1}\right]$ and such that $v_{x}\left(x_{1}\right)=1$. For $x \in\left(x_{1}, x_{0}\right]$ we have $v_{x}<1$ so that (22) fails to be well-defined. By considering the dividend strategy of paying dividends whenever $X_{t}=R_{t}-C_{t}$ exceeds $v\left(x_{1}\right)$, we get indeed that $w(t, x)=e^{-\beta t} v(x)$ is the value function associated to this strategy. But this strategy is not optimal (among all admissible strategies), as it is better to wait with the dividend paying until the process $X$ hits $x_{0}$. 
On the other hand, if $k>k_{0}$ then $k g(x)=v(x)$ satisfies $\inf _{x \geq 0} v_{x}(x)>1$ so that the ODE (22) is satisfied on all of $\mathbb{R}_{\geq 0}$ with the associated optimal dividend strategy being to never ever pay dividends. In economic terms this may be interpreted as a bubble: the process $\left(e^{-\beta t} v\left(X_{t}\right)\right)_{t \geq 0}$ is a local martingale in view of (22). It is not hard to show that it is indeed a martingale, tending almost surely to zero, whence for all $0 \leq t<\infty$ we have $v\left(X_{0}\right)=\mathbb{E}\left[e^{-\beta t} v\left(X_{t}\right)\right]>\mathbb{E}\left[\lim _{t \rightarrow \infty} e^{-\beta t} v\left(X_{t}\right)\right]=0$. This corresponds to the situation of a "pure growth company" whose valuation by the function $w$ although consistent in finite time (this is an economic interpretation of the fact that $\left(e^{-\beta t} v\left(X_{t}\right)\right)_{t \geq 0}$ is a martingale) - is never materialized by actual dividend payments.

After these reminders on the classical case $\alpha=1$ let us discuss the situation $\alpha \in(0,1)$ described in Theorem 1 above. Instead of the ODE (22) we now consider the nonlinear ODE (15); again one initial condition is given by $v(0)=$ 0 , while for the second, we may prescribe a value for $v_{x}(0)$ similarly as above.

If we choose $k=v_{x}(0)>0$ small enough, then it turns out that there is a unique solution of (15) on a maximal interval [0, $\left.x_{1}\right)$, where $0<x_{1}<\infty$. As $x \nearrow x_{1}$ we find that $v_{x}(x)$ tends to zero, so that $v_{x}^{-\frac{\alpha}{1-\alpha}}$ tends to infinity and the ODE (15) does not make sense any more for $x \geq x_{1}$.
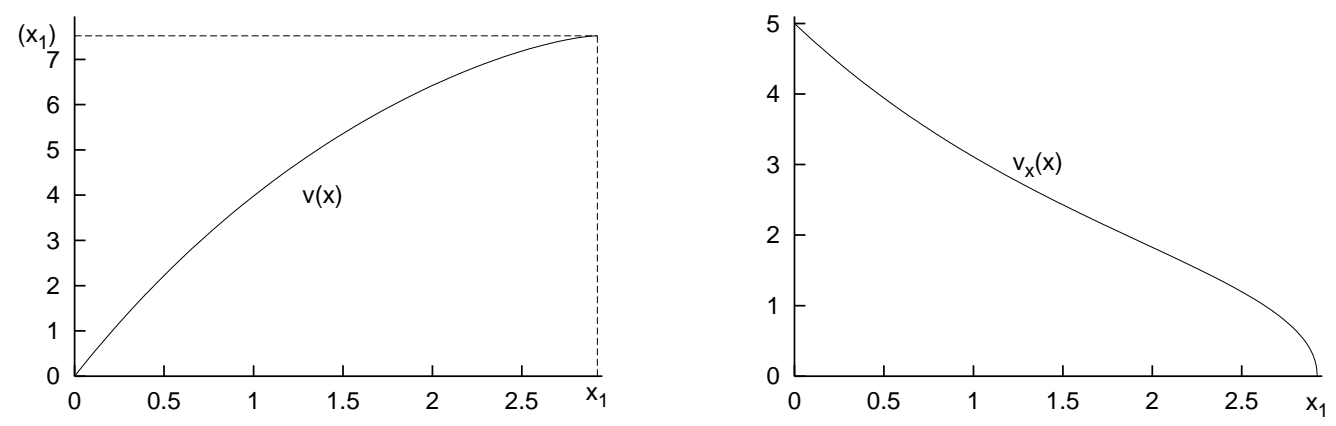

Fig. 2. The functions $v(x)$ and $v_{x}(x)$ with parameters $\alpha=0.5, \beta=0.05, \mu=0.2$, $\sigma=1$, and initial values $v(0)=0$ and $v_{x}(0)=5$.

This function is indeed the value function associated to the dividend policy of paying dividends with an intensity of $v_{x}^{-\frac{1}{1-\alpha}}$. One may check that these dividend payments create a negative drift to the ex-dividend process $X_{t}$, when $X_{t}$ comes close to $v\left(x_{1}\right)$, which is strong enough, such that $X_{t}$ never attains the value $v\left(X_{1}\right)$ a.s. (we assume $X_{0}<v\left(x_{1}\right)$ ). One may also show that this dividend policy is optimal among all dividend policies satisfying the constraint that the ex-dividend process $X_{t}$ remains below $v\left(X_{1}\right)$. The crux is, of course, that this is an artificial constraint and that this policy is not optimal among 
all the admissible dividend payment plans.

On the other hand, if we choose $k=v_{x}(0)>0$ big enough, then $v_{x}>0$ on all of $\mathbb{R}_{\geq 0}$, and there is a unique inflection point $0<x_{2}<\infty$ such that $v$ is concave on $\left[0, x_{2}\right]$ and convex on $\left[x_{2}, \infty\right)$, similarly as in the case $\alpha=1$ considered above.
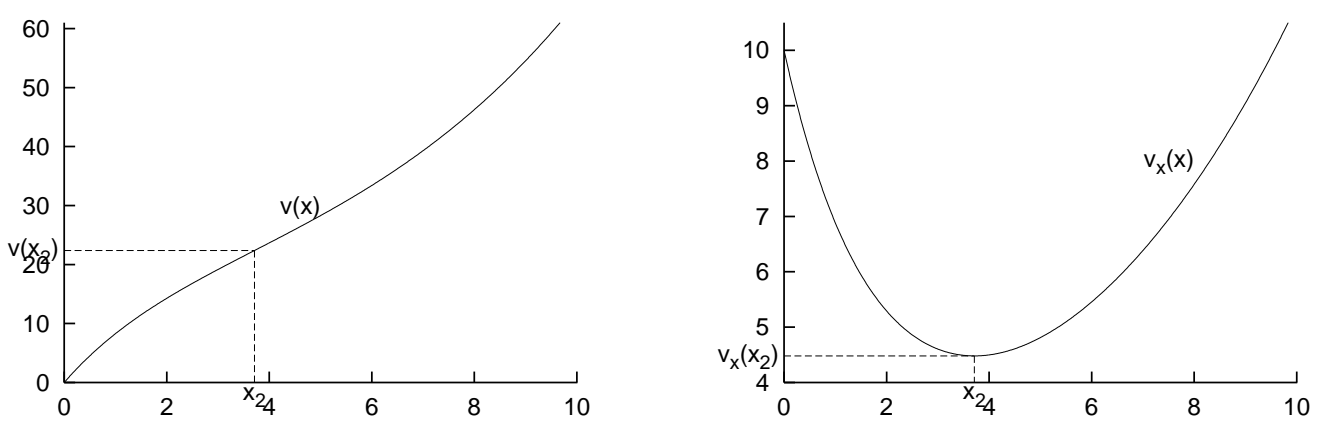

Fig. 3. The functions $v(x)$ and $v_{x}(x)$ with parameters $\alpha=0.5, \beta=0.05, \mu=0.2$, $\sigma=1$, and initial values $v(0)=0$ and $v_{x}(0)=10$.

Economically speaking, this again corresponds to a bubble, caused by a believe in the value of the company not sufficiently materialized by dividend payments in finite time: indeed, as $x \rightarrow \infty$ we have $\lim _{x \rightarrow \infty} v_{x}(x)=\infty$; hence the intensity $v_{x}^{-\frac{1}{1-\alpha}}$ of dividend payments tends to zero when the ex-dividend process $X$ tends to infinity, which leads to a similar phenomenon as in the above discussed case $\alpha=1$.

Hence we have seen that by choosing $k=v_{x}(0)$ either too small or too big does not yield the optimal solution. It turns out - this is the message of Theorem 1 - that there is precisely one critical value $k_{0}=v_{x}(0)$ such that the corresponding solution of (15) is defined on all of $\mathbb{R}_{\geq 0}$ and such that there is no inflection point; hence $v$ is concave on all of $\mathbb{R}_{\geq 0}$ and Theorem 1 furnishes more precise information.

The present choice of $k_{0}$ is similar to the above discussed critical choice in the case $\alpha=1$. There is, however, a remarkable difference: in the case $\alpha=1$ the associated value function $v$ was obtained by some "smooth pasting" at the point $x_{0}$, where the behavior of $v$ changed from that of a solution to (22) to that of a straight line. In the setting, where $\alpha \in(0,1)$, no such "pasting" takes place and $v$ equals the solution to (15) on all of $\mathbb{R}_{\geq 0}$.

Finally we want to discuss the limiting behavior of the situation described by Theorem 1 as $\alpha$ tends to 1 .

Theorem 2 Let $w_{\alpha}(t, x)=e^{-\beta t} v_{\alpha}(x)$ denote the value function associated to 
problem (10) for $\alpha \in(0,1]$ and suppose $\mu>0$. Then

$$
\begin{array}{ll}
\lim _{\alpha \rightarrow 1} v^{(\alpha)}(x)=v^{(1)}(x) & (x \geq 0) . \\
\lim _{\alpha \rightarrow 1} v_{x}^{(\alpha)}(x)=v_{x}^{(1)}(x) & (x \geq 0),
\end{array}
$$

the convergence being uniform on compact subsets of $[0,+\infty)$. Moreover

$$
\lim _{\alpha \rightarrow 1} c^{(\alpha)}(x)=\left\{\begin{array}{l}
0, \quad\left(0 \leq x<x_{0}\right), \\
+\infty, \quad\left(x>x_{0}\right) .
\end{array}\right.
$$

There is a remarkable feature in this theorem: we just observed that $v^{(1)}$ is a "pasted" solution, which has a discontinuity of its third derivative $v_{x x x}^{(1)}$ at the point $x_{0}$. On the other hand, the functions $v^{(\alpha)}$ are not "pasted", i.e., solutions to (15) and therefore infinitely often differentiable on all of $\mathbb{R}_{\geq 0}$.

This gives us some motivation, why we should not expect to find nice closedform solutions to the ODE (15). A precise statement in this context may be formulated like that: there does not exist a domain $\mathcal{D}$ in $\mathbb{C}$, containing $x_{0}$ as an interior point, such that the functions $v^{(\alpha)}$ can all be analytically continued to $\mathcal{D}$, and such that $\left(v^{(\alpha)}\right)_{0<\alpha<1}$ is a normal family, see (Rudin, 1987, Ch.14, p.281). Indeed, otherwise the pointwise (on $\mathbb{R}_{\geq 0}$ ) limit $v^{(1)}$ (of a convergent subsequence) would also have an analytic continuation to $\mathcal{D}$, which is not true in view of the discontinuity of the third derivative, (Rudin, 1987, Ch.10, p.214).

We close this introduction with a remark on more general utility functions $U(x)$ than just $U(x)=\frac{x^{\alpha}}{\alpha}$, which has been pointed out to us by N. Touzi. Under the general assumptions on $U$ made above, we may still write the HJB equation (11):

$$
w_{t}+\sup _{c \geq 0}\left[(\mu-c) w_{x}+\frac{\sigma^{2}}{2} w_{x x}+U(c) e^{-\beta t}\right]=0, \quad w(t, 0)=0 .
$$

Denote by $V(y)$ the conjugate function of $U(x)$ (see, e.g., Rockafellar (1970), and Karatzas et al. (1991) or Kramkov and Schachermayer (1999) for its applications in the context of finance)

$$
V(y)=\sup _{x \geq 0}[U(X)-x y]
$$


Note that, for $U(x)=\frac{x^{\alpha}}{\alpha}$ we have $V(y)=\frac{1-\alpha}{\alpha} y^{-\frac{\alpha}{1-\alpha}}$. Using again $w_{t}=-\beta w$ we therefore obtain, also for more general utility functions, the ODE

$$
-\beta v+\mu v_{x}+\frac{\sigma^{2}}{2} v_{x x}+V\left(v_{x}\right)=0
$$

This equation makes the present problem — at least in principle - also approachable by ODE methods and gives additional insight on the term $-\frac{1-\alpha}{\alpha} v_{x}^{-\frac{\alpha}{1-\alpha}}$ in $(15)$.

The remainder of the paper is organized as follows: In Section 2 we verify that $w(t, x)=e^{-\beta t} v(x)$ as defined in Theorem 1 is indeed the value function and construct the corresponding optimal dividend strategy. In Section 3 we provide results about the ODE (15), with a few technical results relegated to Appendices A and B.

\section{Verification}

In Proposition 1 below it is shown that the ODE (15) has a solution $v(x)$ with properties as stated in Theorem 1 . The asymptotic results as $x \rightarrow \infty$ are shown by Proposition 2. In this section we show - admitting Proposition 1 - that the function

$$
w(t, x)=e^{-\beta t} v(x)
$$

is indeed the value function defined in (10), with the optimal control uniquely attained at the dividend strategy $C$, that is constructed as follows: The case $x=0$ is trivial, as it implies $C_{t}=0$ for $t \geq 0$. Suppose $x>0$ and define the function $c$ on $\mathbb{R}_{>0}$ by

$$
c(x)=v_{x}(x)^{-\frac{1}{1-\alpha}}, \quad(x>0) .
$$

Standard arguments show that there is a maximal stopping time $\tau>0$, such that the stochastic differential equation

$$
d X_{t}=\left(\mu-c\left(X_{t}\right)\right) d t+\sigma d W_{t}, \quad X_{0}=x,
$$

admits a unique strong solution on $[0, \tau)$. This solution is continuous, strictly positive on $[0, \tau)$, and satisfies $\lim _{t \rightarrow \tau} X_{t}=0$ almost surely. We set $X_{t}=0$ for 
$t \geq \tau$. Then $\tau=\inf \left\{t \geq 0: X_{t}=0\right\}$. Now we can define the process

$$
c_{t}= \begin{cases}c\left(X_{t}\right) & (0 \leq t<\tau) \\ 0 & (t \geq \tau)\end{cases}
$$

and

$$
C_{t}=\int_{0}^{t} c_{s} d s, \quad(t \geq 0) .
$$

Lemma 1 Given any nonnegative adapted process $\left(c_{t}\right)_{t \geq 0}$ such that the process $C_{t}=\int_{0}^{t} c_{s} d s$ of accumulated dividend payments is admissible, we have, that the process

$$
Z_{t}=w\left(t, X_{t \wedge \tau}\right)+\int_{0}^{t} e^{-\beta s} U\left(c_{s}\right) d s, \quad t \geq 0,
$$

is a nonnegative supermartingale, where $X_{t}=R_{t}-C_{t}$ and $\tau=\inf \{t \geq 0$ : $\left.X_{t}=0\right\}$. Hence

$$
v\left(X_{0}\right) \geq \mathbb{E}\left[\int_{0}^{\infty} e^{-\beta s} U\left(c_{s}\right) d s\right] .
$$

If $\left(c_{t}\right)_{t \geq 0}$ is admissible, but different from the process defined in (35), then we have a strict inequality in (38).

Proof: By Itô's Lemma we have

$$
\begin{aligned}
d Z_{t}= & w_{t}\left(t, X_{t}\right) d t+w_{x}\left(t, X_{t}\right) d X_{t}+\frac{1}{2} w_{x x}\left(t, X_{t}\right) d\langle X\rangle_{t}+e^{-\beta t} U\left(c_{t}\right) d t \\
= & {\left[w_{t}\left(t, X_{t}\right)+\left(\mu-c_{t}\right) w_{x}\left(t, X_{t}\right)+\frac{\sigma^{2}}{2} w_{x x}\left(t, X_{t}\right)+e^{-\beta t} U\left(c_{t}\right)\right] d t } \\
& +\sigma w_{x}\left(t, X_{t}\right) d W_{t} .
\end{aligned}
$$

Whence equation (11) shows that $Z$ is a local supermartingale; as $Z \geq 0$, it is a supermartingale. Hence, for any starting point $X_{0} \geq 0$ and $t \geq 0$

$$
v\left(X_{0}\right) \geq \mathbb{E}\left[w\left(t, X_{t}\right)+\int_{0}^{t} e^{-\beta t} U\left(c_{s}\right) d s\right]
$$




$$
\geq \mathbb{E}\left[\int_{0}^{t} e^{-\beta s} U\left(c_{s}\right) d s\right]
$$

Letting $t \rightarrow \infty$ and applying the monotone convergence theorem we obtain the result.

When $c$ is different from the process defined in (35), in the sense that the subset of $\Omega \times \mathbb{R}_{\geq 0}$, where the processes differ has strictly positive $P \times \lambda$ measure, with $\lambda$ the Lebesgue measure on $\mathbb{R}_{\geq 0}$, then we have by the strict concavity of $U$ and by (11)

$$
d Z_{t}=\mu_{t} d t+\sigma w_{x}\left(t, X_{t}\right) d W_{t}
$$

where $\mu$ is a non-positive process different from zero, in the sense defined above. Hence $Z_{0}>\mathbb{E}\left[Z_{\infty}\right]$, which yields a strict inequality in (41).

Lemma 2 For $w, c$, and $X$ as defined by (32), (34), and (35) above we have that

$$
Z_{t}=w\left(t, X_{t}\right)+\int_{0}^{t} e^{-\beta s} U\left(c_{s}\right) d s, \quad t \geq 0,
$$

is a martingale and that

$$
\lim _{t \rightarrow \infty} \mathbb{E}\left[w\left(t, X_{t}\right)\right]=0
$$

Hence

$$
Z_{0}=v\left(X_{0}\right)=\mathbb{E}\left[\int_{0}^{\infty} e^{-\beta s} U\left(c_{s}\right) d s\right] .
$$

Proof: By the same argument as in Lemma 1 we now obtain that $Z$ is a local martingale, see (15) and (39). We have to show that it is a true martingale as well as equation (44) which will clearly imply (45).

So, let us show that, for every fixed horizon $T<\infty$, the process $\left(Z_{t}\right)_{0 \leq t \leq T}$ is uniformly integrable. To do so, it will suffice to show, that the maximal function $Z_{T}^{*}=\sup _{0 \leq t \leq T} Z_{t}$ is integrable. We have

$$
0 \leq Z_{T}^{*} \leq \sup _{0 \leq t \leq T}\left[v\left(X_{t}\right)\right]+\int_{0}^{T} e^{-\beta t} U\left(c_{t}\right) d t
$$


The second term on the right hand side is integrable by (38), and for the first term we can argue by the following rough estimate: we have $X_{t} \leq R_{t}$ and $v(x) \leq x v^{\prime}(0)$ by the concavity of $v$. Hence

$$
\mathbb{E}\left[\sup _{0 \leq t \leq T} v\left(X_{t}\right)\right] \leq v^{\prime}(0) \mathbb{E}\left[\sup _{0 \leq t \leq T} R_{t}\right]<\infty .
$$

The second inequality of (47) holds true as $R$ is simply Brownian motion with drift. Summing up, we have shown that $\left(Z_{t}\right)_{0 \leq t \leq T}$ is a true martingale. In particular, for each $0<t<\infty$

$$
v\left(X_{0}\right)=Z_{0}=\mathbb{E}\left[w\left(t, X_{t}\right)\right]+\mathbb{E}\left[\int_{0}^{t} e^{-\beta s} U\left(c_{s}\right) d s\right]
$$

We now show (44): Let $g(x)=e^{\lambda_{1} x}-e^{\lambda_{2} x}$, where $\lambda_{1}>0$ and $\lambda_{2}<0$ are given by (24). Thus $g$ is a solution to (23) on $\mathbb{R}_{\geq 0}$. We know that $\left(e^{-\beta t} g\left(R_{t}\right)\right)_{t>0}$ is a local martingale, hence, by the monotonicity of $g$, the process $\left(e^{-\beta t} g\left(X_{t}\right)\right)_{t \geq 0}$ is a local supermartingale and therefore, by the nonnegativity of $g$, a supermartingale. Hence

$$
\mathbb{E}\left[e^{-\beta t} g\left(X_{t}\right)\right] \leq g\left(X_{0}\right), \quad \text { for } t \geq 0
$$

Note that $\lim _{x \rightarrow \infty} v(x) / g(x)=0$ so that, for $\varepsilon>0$, we may find $t_{\varepsilon}>0$ such that

$$
e^{-\beta t} v(x) \leq \varepsilon \max \left[1, e^{-\beta t} g(x)\right] \quad \text { for } x \geq 0, t \geq t_{\varepsilon} .
$$

Hence, for $t \geq t_{\varepsilon}$,

$$
\mathbb{E}\left[e^{-\beta t} v\left(X_{t}\right)\right] \leq \varepsilon\left(1+\mathbb{E}\left[e^{-\beta t} g\left(X_{t}\right)\right]\right) \leq \varepsilon\left(1+g\left(X_{0}\right)\right),
$$

which proves (44).

Combining (48) and (44) and letting $t \rightarrow \infty$ we obtain (45) from the monotone convergence theorem.

The above argument also shows the classical result that, for the case $\alpha=1$, the function $v^{(1)}$ is indeed the value function of the problem (3) and that the corresponding dividend policy of paying dividends, when $X$ hits $x_{0}$, is indeed optimal. In fact, the argument used in Lemma 2 becomes somewhat easier, as now the process $X$ is bounded and therefore trivially uniformly integrable. 
We can also apply the above arguments for $0<\alpha<1$, to the solutions of the ODE (15) where we choose $v_{x}(0)$ either smaller or bigger than the critical value $k_{0}$.

In the former case, denote again by $x_{1}>0$ the maximal value such that $v$ is defined and satisfies (15) on $\left[0, x_{1}\right)$ and let the dividend strategy be defined via (35). Then Lemma 2 still holds true and Lemma 1 holds true for all dividend policies $c$ such that $X$ remains a.s. in $\left[0, x_{1}\right.$ ) (with almost identical arguments). In plain words: the dividend policy is optimal among all policies such that the ex-dividend process never exceeds $x_{1}$. But this is, of course, an artificial constraint, lowering the value function.

In the latter case, where $v_{x}(0)>x_{1}$, Lemma 1 still is satisfied (without restrictions). In Lemma 2 the process $Z$ still is a martingale; but (44) and therefore (45) fail to hold true. An easy and direct argument to see the failure of (44) goes as follows: Note that in the present case $\inf _{x>0} v_{x}(x)>0$ so that

there is a (deterministic) uniform upper bound for $c_{t}=v_{x}\left(X_{t}\right)^{-\frac{1}{1-\alpha}}$ as well as $\int_{0}^{\infty} e^{-\beta s} U\left(c_{s}\right) d s$. It follows immediately that (45) cannot hold true for large values of $X_{0}$. To extend this reasoning to arbitrary initial values $X_{0}>0$ it suffices to condition on the event that at some fixed time $t>0$ (say, $t=1)$ the process $X$ satisfies $X_{t} \geq M$, for some large $M$. This implies that the conditional version of (45) fails, which easily implies - using the supermartingale property of $Z$ - that (45) is wrong too. This phenomenon may again be interpreted as a "bubble" similarly as in the case $\alpha=1$ discussed above.

\section{Results about the ODE}

\subsection{Existence and uniqueness}

Proposition 1 Suppose $\alpha \in(0,1), \beta>0, \mu \in \mathbb{R}, \sigma>0$. Then

$$
v(0)=0, \quad \beta v-\mu v_{x}-\frac{\sigma^{2}}{2} v_{x x}-\frac{1-\alpha}{\alpha} v_{x}^{-\frac{\alpha}{1-\alpha}}=0, \quad(x \geq 0)
$$

has precisely one strictly increasing concave solution in $C^{(2)}[0, \infty)$.

Proof: We have a second order nonlinear equation, with the independent variable not in the equation. By Riccati's Ansatz the problem is equivalent to two first order equations: Suppose we have a strictly increasing concave solution $v \in C^{(2)}[0, \infty)$. Then $v(0)>0$ for all $x>0$ and $v_{x}(x)>0$ for all $x \geq 0$. We 
can define $y(x)=v_{x}\left(v^{-1}(x)\right)$ for all $x \geq 0$. The functions $v$ and $y$ satisfy

$$
v(0)=0, \quad v_{x}=y(v), \quad(x \geq 0)
$$

and

$$
y_{x}=\frac{2}{\sigma^{2}}\left(\frac{\beta x}{y}-\mu-\frac{1-\alpha}{\alpha} y^{-\frac{1}{1-\alpha}}\right), \quad(x \geq 0) .
$$

We have $y(x)>0$ for all $x \geq 0$. From $v_{x x}=y_{x}(v(x)) v_{x}(x)$ for all $x \geq 0$ and the concavity of $v$ it follows, that $y_{x}(x) \leq 0$ for all $x \geq 0$, thus $y$ is decreasing, strictly positive and hence bounded. The right hand sides of (53) and (54) satisfy Lipschitz conditions on compact subsets of $[0, \infty) \times(0, \infty)$. As a consequence we have uniqueness.

Theorem 3 below asserts the existence of a decreasing, strictly positive, bounded solution of (54), that is Lipschitz continuous on compact subsets of $(0, \infty)$. This implies by a standard argument the existence of a solution to (53), with properties as claimed.

It is convenient for the proofs below, to study the ODE (54) on $x \in \mathbb{R}$ instead of $x \geq 0$.

Theorem 3 Suppose $\alpha \in(0,1), \beta>0, \mu \in \mathbb{R}, \sigma>0$. Then the differential equation

$$
y_{x}=\frac{2}{\sigma^{2}}\left(\frac{\beta x}{y}-\mu-\frac{1-\alpha}{\alpha} y^{-\frac{1}{1-\alpha}}\right), \quad(x \in \mathbb{R})
$$

has precisely one decreasing convex solution.

Proof: In order to keep the argument transparent, we only give an outline of the proof. The technical details are done in various lemmas which have been shifted to the appendix. (i) First we study potential critical points of $y(x)$, that is, solutions of $F(x, y)=0$ with $x \in \mathbb{R}$ and $y>0$, where

$$
F(x, y)=\frac{2}{\sigma^{2}}\left(\frac{\beta x}{y}-\mu-\frac{1-\alpha}{\alpha} y^{-\frac{1}{1-\alpha}}\right) .
$$

They can be parameterized by $\{x=\zeta(y): y>0\}$. The function $\zeta$ is described in Lemma 5. Alternatively the potential critical points can be described by $\left\{y=\bar{\theta}(x): x \geq \hat{x}_{1}\right\}$ and $\left\{y=\underline{\theta}(x): x \geq \hat{x}_{1}\right\}$, with some $\hat{x}_{1} \geq 0$. The value $\hat{x}_{1}$ is given in Lemma 5 and functions $\bar{\theta}$ and $\underline{\theta}$ are described in Lemma 6 . 


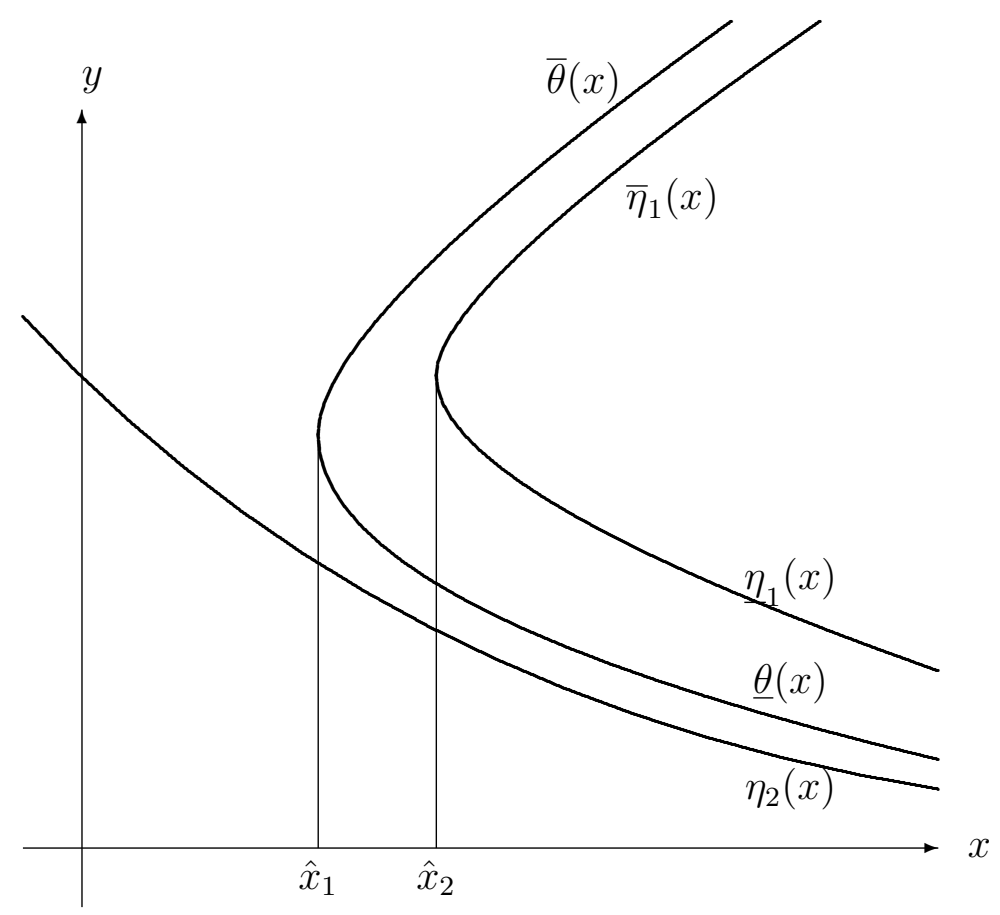

Fig. 4. A sketch for the auxiliary functions $\bar{\theta}, \underline{\theta}, \bar{\eta}_{1}, \underline{\eta}_{1}, \eta_{2}$.

(ii) Next we discuss potential inflection points of $y$. As

$$
\begin{aligned}
y_{x}(x) & =F_{x}(x, y(x))+F_{y}(x, y(x)) y_{x}(x) \\
& =F_{x}(x, y(x))+F_{y}(x, y(x)) F(x, y(x)),
\end{aligned}
$$

those are the solutions of $G(x, y)=0$ with $x \in \mathbb{R}$ and $y>0$, where

$$
G(x, y)=F_{x}(x, y)+F_{y}(x, y) F(x, y)
$$

They can be parameterized by $\left\{x=\xi_{1}(y): y>0\right\}$ and $\left\{x=\xi_{2}(y): y>0\right\}$. The functions $\xi_{1}$ and $\xi_{2}$ are studied in Lemma 7 . Alternatively the potential inflections points are described by $\left\{y=\bar{\eta}_{1}(x): x \geq \hat{x}_{2}\right\},\left\{y=\underline{\eta}_{1}(x): x \geq \hat{x}_{2}\right\}$, and $\left\{y=\eta_{2}(x): x \in \mathbb{R}\right\}$, with some $\hat{x}_{2}>0$. The value $\hat{x}_{2}$ is given and the functions $\bar{\eta}_{1}, \underline{\eta}_{1}$, and $\eta_{2}$ are described in Lemma 9 , with an auxiliary result in Lemma 8. This discussion of auxiliary functions is illustrated with Figure 4. The functions $\underline{\theta}$ and $\eta_{2}$ will be used to construct the desired solution $y$ of the ODE (55). They provide good upper and lower bounds for $y(x)$ when $x$ is large. From now on $\underline{\theta}$ and $\eta_{2}$ will be denoted by $\theta$ and $\eta$.

(iii) In Lemma 11 and 12 we show that

$$
y(b)=\theta(b), \quad y_{x}=F(x, y), \quad(x \in \mathbb{R})
$$


has a unique solution for any $b>\hat{x}_{1}$. This solution will be denoted by $\bar{y}_{b}$. In Lemma 13 and 14 we show that for each $b>0$ there is $r_{b}>0$ such that

$$
y(b)=\eta(b), \quad y_{x}=F(x, y), \quad\left(x \leq r_{b}\right)
$$

has a unique solution that explodes down to zero as $x \rightarrow r_{b}$, while the first derivative tends to $-\infty$ as $x \rightarrow r_{b}$. Those solutions will be denoted by $\underline{y}_{b}$. Moreover we obtain the inequalities

$$
\eta(x)<\underline{y}_{b}(x), \quad(x \leq b)
$$

and

$$
\bar{y}_{b}(x)<\theta(x), \quad\left(\hat{x}_{1} \leq x \leq b\right) .
$$

(iv) By construction $\bar{y}_{b}(x)$ is bounded and decreasing in $b$, whereas $\underline{y}_{b}(x)$ is bounded and increasing in $b$. Hence we can define for all $x \in \mathbb{R}$ the limits

$$
\bar{y}_{\infty}(x)=\lim _{b \rightarrow \infty} \bar{y}_{b}(x), \quad \underline{y}_{\infty}(x)=\lim _{b \rightarrow \infty} \underline{y}_{b}(x) .
$$

Both $\bar{y}_{\infty}$ and $\underline{y}_{\infty}$ are positive-valued, decreasing, convex, and continuous. Moreover $\underline{y}_{\infty}(x) \leq \bar{y}_{\infty}(x)$ for all $x \in \mathbb{R}$. In Lemma 15 we show that $\bar{y}_{\infty}=\underline{y}_{\infty}$. From now on we will call this function just $y$. Moreover, using Dini's Theorem, we show that $y$ is a solution of (55) with the desired properties, and that it is unique. The various solutions and bounds are illustrated in Figure 5. 


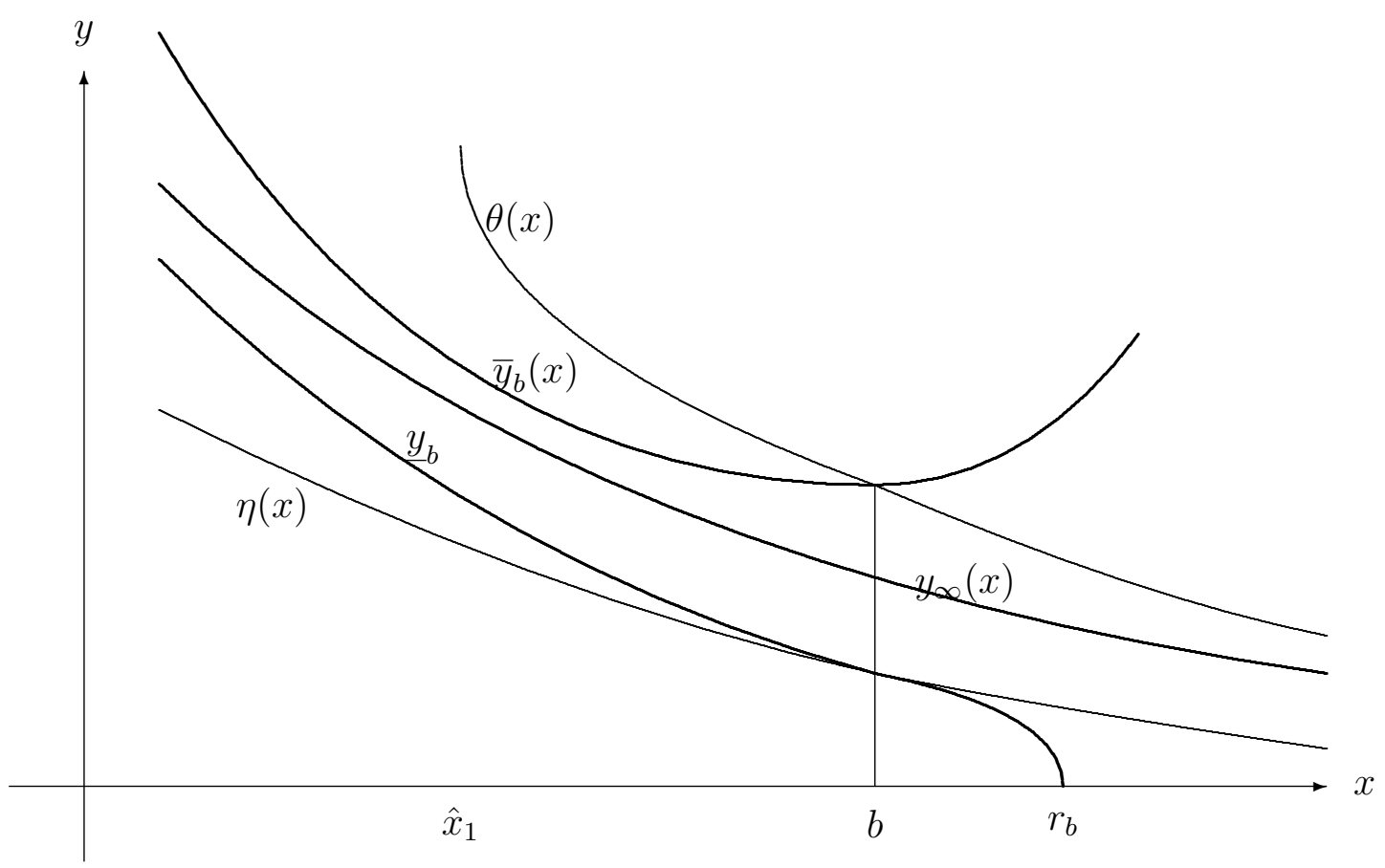

Fig. 5. Sketch of $\bar{y}_{b}, \underline{y}_{b}$, the solution $y_{\infty}$, and the bounds $\theta$ and $\eta$.

(v) The function $y$ is nonnegative, decreasing, and smooth. As a consequence it is locally Lipschitz and bounded, hence a standard theorem on existence and uniqueness of the solution of (15).

(vi) By construction we have

$$
\eta(x) \leq y(x) \leq \theta(x), \quad\left(x \geq \hat{x}_{1}\right) .
$$

The leading terms of $\theta(x)$ and $\eta(x)$ for $x \rightarrow \infty$ coincide and provide the exact order of magnitude of $y(x)$ as $x \rightarrow \infty$. Using standard comparison theorems for ODEs, we can lift in Proposition 2 those asymptotic developments to $v(x)$ as $x \rightarrow \infty$.

Before we proceed with the proof of Theorem 2, we indicate, why it would be surprising, if the ODE (54) had an elementary solution: Particular simple cases should be $\alpha=n /(n+1)$ with integer $n \geq 1$, because the right hand side of the ODE is then a rational function of $x$ and $y$, which should make life easier. (Recall that one of our aims is to analyze the limiting behavior as $\alpha \rightarrow 1$.) Reducing further to simple cases consider the following choice of parameters: $\alpha=1 / 2$, and we may take $\beta=1, \mu=0, \sigma=\sqrt{2}$. Then we have the harmless looking ODE

$$
y^{\prime}=\frac{x}{y}-\frac{1}{y^{2}} \text {. }
$$


Interchanging the roles of the variables yields

$$
(x y-1) x^{\prime}-y^{2}=0,
$$

an Abel ODE of the second kind and class C (see Kamke, 1977, p.28). And we have found neither by using Maple nor checking the literature an elementary solution for that.

\subsection{Proof of Theorem 2}

As in the initial discussion following Theorem 1 , let $v^{(1)}$ denote the value function for the case $\alpha=1$, that is,

$$
v^{(1)}(x)= \begin{cases}k_{0}\left(e^{\lambda_{1} x}-e^{\lambda_{2} x}\right), & \left(0 \leq x \leq x_{0}\right), \\ \frac{\mu}{\beta}-x_{0}+x, & \left(x \geq x_{0}\right) .\end{cases}
$$

with

$$
\lambda_{1}=\frac{-\mu+\sqrt{\mu^{2}+2 \beta \sigma^{2}}}{\sigma^{2}}, \quad \lambda_{2}=\frac{-\mu-\sqrt{\mu^{2}+2 \beta \sigma^{2}}}{\sigma^{2}},
$$

and

$$
x_{0}=\frac{2}{\lambda_{1}-\lambda_{2}} \ln \left(-\frac{\lambda_{2}}{\lambda_{1}}\right), \quad k_{0}=\frac{1}{\lambda_{1} e^{\lambda_{1} x_{0}}-\lambda_{2} e^{\lambda_{2} x_{0}}} .
$$

Then $v^{(1)}$ is the unique solution to the problem

$$
v\left(x_{0}\right)=\frac{\mu}{\beta}, \quad v_{x}\left(x_{0}\right)=1, \quad v_{x x}=-\frac{2}{\sigma^{2}}\left(\mu v_{x}-\beta v\right)_{+}, \quad(x \geq 0) .
$$

Uniqueness follows immediately from a Lipschitz condition of the right hand side. Let

$$
y^{(1)}(x)=v_{x}^{(1)}\left(\left(v^{(1)}\right)^{-1}(x)\right),
$$

where $\left(v^{(1)}\right)^{-1}$ denotes the inverse function of $v^{(1)}$. 
Lemma 3 Suppose we have differentiable functions $y$ and $v$ that satisfy

$$
y\left(\frac{\mu}{\beta}\right)=1, \quad y_{x}=-\frac{2}{\sigma^{2}}\left(\mu-\frac{\beta x}{y}\right)_{+}, \quad(x \geq 0),
$$

and

$$
v(0)=0, \quad v_{x}=y(v), \quad(x \geq 0),
$$

then $y=y^{(1)}$ and $v=v^{(1)}$.

Proof: A direct calculation shows that $y^{(1)}$ and $v^{(1)}$ satisfy (73) resp. (74). From (73) it follows, that any solution $y$ is decreasing and satisfies $y(x) \geq 1$ for $x \geq 0$. The right hand side is locally Lipschitz on $\mathbb{R}_{+}^{2}$, thus we have uniqueness. Similarly the right hand side of (74) is locally Lipschitz and thus there is uniqueness for $v$.

Lemma 4 We have

$$
\lim _{\alpha \rightarrow 1} y^{(\alpha)}(x)=y^{(1)}(x), \quad(x \geq 0),
$$

and the convergence is uniform on compact subsets of $[0,+\infty)$.

Proof: (i) We consider an arbitrary sequence $\left(\alpha_{n}\right)$ with $\alpha_{n} \in(0,1)$ and $\alpha_{n} \rightarrow 1$ as $n \rightarrow \infty$. We fix arbitrary $a<0$ and $b>0$. We know from Theorem 3 that $\left(y^{\left(\alpha_{n}\right)}\right)$ is a sequence of decreasing convex functions. Equation (B.46) in Lemma 22 shows that that $y^{\left(\alpha_{n}\right)}(x)$ is uniformly bounded on $[a, b]$ as $n \rightarrow \infty$. By Helly's selection principle (Helly, 1912, §6.VII.5, p.286), see also (Feller, 1971, VIII.6), we can find a subsequence $\left(\alpha_{n}^{\prime}\right)$, such that $y^{\left(\alpha_{n}^{\prime}\right)}$ converges weakly to a limit $y$. Weak convergence in this context implies, that we have convergence at the continuity points of the limit. But this limit $y$ is a limit of convex functions, thus convex, and therefore continuous on $(a, b)$. Hence we have in fact pointwise convergence on $(a, b)$.

Consider now an arbitrary $a^{\prime} \in(a, 0)$ and $b^{\prime} \in(0, b)$. Then our subsequence provides a sequence of monotonic functions that converges to a continuous limit on the compact interval $\left[a^{\prime}, b^{\prime}\right]$. By a complement to Dini's Theorem we must have uniform convergence on $\left[a^{\prime}, b^{\prime}\right]$, see (Pólya and Szegö, 1925, II.3.3.127, pp.63 and 226).

(ii) We know from equation (B.45) in Lemma 22, that $y^{(\alpha)}(x)$ is uniformly bounded away from zero on $\left[a^{\prime}, b^{\prime}\right]$, as $\alpha \rightarrow 1$. Thus

$$
\lim _{n \rightarrow \infty} \frac{x}{y^{\left(\alpha_{n}^{\prime}\right)}(x)}=\frac{x}{y(x)}
$$


uniformly on $\left[a^{\prime}, b^{\prime}\right]$. Equation (B.39) in Lemma 21 says

$$
\lim _{n \rightarrow \infty} \frac{1-\alpha}{\alpha} y^{\left(\alpha_{n}^{\prime}\right)}(x)^{-\frac{1-\alpha}{\alpha}}=0, \quad\left(0 \leq \frac{\mu}{\beta}\right)
$$

uniformly. Thus $y_{x}^{\left(\alpha_{n}^{\prime}\right)}$ is uniformly convergent and

$$
\lim _{n \rightarrow \infty} y_{x}^{\left(\alpha_{n}^{\prime}\right)}(x)=y_{x}(x)
$$

This shows that $y$ solves (73) and thus $y=y^{(1)}$. By these arguments we see, that $y^{(\alpha)}$ has exactly one accumulation point, thus

$$
\lim _{\alpha \rightarrow 1} y^{(\alpha)}(x)=y^{(1)}(x) .
$$

Again, by referring to the complement to Dini's Theorem, this limit is uniform on compacts.

Now we are ready to complete the proof of Theorem 2: Let us consider the problem

$$
v(0)=0, \quad v^{\prime}=y^{(\alpha)}(v), \quad(x \geq 0) .
$$

The functions $p^{(\alpha)}$ are continuous and converge uniformly on compact subsets of $[0,+\infty)$ as $\alpha \rightarrow 1$ to $y^{(1)}$, which is locally Lipschitz. Let $v^{(\alpha)}$ denote the solution of (80). Then we can apply a standard theorem about the continuity of solutions of ODEs with respect to a parameter, see, for example, the corollary in Section 6.4, p.157 of Birkhoff and Rota (1969). The conclusion is, that $v^{(\alpha)}$ converges to a solution of $(74)$, which is precisely $v^{(1)}$. As we have from the ODE (74)

$$
v_{x}^{(\alpha)}(x)=y^{(\alpha)}\left(v^{(\alpha)}(x)\right)
$$

and both $y^{(\alpha)} \rightarrow y^{(1)}$ and $v^{(\alpha)} \rightarrow v^{(1)}$ uniformly on compact subsets of $[0, \infty)$, we get $v_{x}^{(\alpha)}(x) \rightarrow v_{x}^{(1)}(x)$ uniformly on compact subsets of $[0, \infty)$ as well. Next suppose $x<x_{0}$, then $v_{x}^{(\alpha)}(x) \rightarrow v_{x}^{(1)}(x)>1$ and thus

$$
\lim _{\alpha \rightarrow 1} v_{x}^{(\alpha)}(x)^{-\frac{1}{1-\alpha}} \rightarrow 0, \quad\left(0 \leq x<x_{0}\right) .
$$

Finally if $x>x_{0}$ then using again the uniform convergence of $v^{(\alpha)}(x) \rightarrow$ $v^{(1)}(x)>\mu / \beta+x-x_{0}$ and Lemma 21 yields

$$
\lim _{\alpha \rightarrow 1} \frac{1-\alpha}{\alpha}\left(y^{(\alpha)}\left(v^{(\alpha)}(x)\right)\right)^{-\frac{1}{1-\alpha}}=\beta\left(x-x_{0}\right)_{+}>0, \quad\left(x>x_{0}\right)
$$


implying

$$
\lim _{\alpha \rightarrow 1}\left(y^{(\alpha)}\left(v^{(\alpha)}(x)\right)\right)^{-\frac{1}{1-\alpha}}=+\infty, \quad\left(x>x_{0}\right)
$$

This proves (28).

\section{Acknowledgments}

We thank N. Touzi and S. Asmussen for helpful discussions and comments.

\section{A Appendix: Study of the nonlinear ODE}

We consider the ODE

$$
y_{x}=F(x, y), \quad(x \in \mathbb{R}),
$$

with

$$
F(x, y)=\frac{2}{\sigma^{2} y}\left(\beta x-\frac{1-\alpha}{\alpha} y^{-\frac{\alpha}{1-\alpha}}-\mu y\right) .
$$

We will use later

$$
F_{x}(x, y)=\frac{2 \beta}{\sigma^{2} y}
$$

and

$$
F_{y}(x, y)=\frac{2}{\sigma^{2} y^{2}}\left(\frac{1}{\alpha} y^{-\alpha /(1-\alpha)}-\beta x\right) .
$$

\section{A.1 Critical points}

It is useful to look at $(x, y)$ where $F(x, y)$ vanishes.

Lemma 5 (i) The solutions of $F(x, y)=0$ with $(x, y) \in \mathbb{R} \times \mathbb{R}_{>0}$ can be parameterized by $x=\zeta(y), y>0$, where $\zeta(y)$ is the function given by

$$
\zeta(y)=\frac{\mu}{\beta} y+\frac{1-\alpha}{\alpha \beta} y^{-\frac{\alpha}{1-\alpha}}, \quad y>0 .
$$


(ii) Let

$$
\hat{x}_{1}=\frac{\mu_{+}^{\alpha}}{\alpha \beta}, \quad \hat{y}_{1}= \begin{cases}\left(\frac{-\mu \alpha}{1-\alpha}\right)^{-(1-\alpha)} & \text { if } \mu<0, \\ +\infty & \text { if } \mu=0, \\ \mu^{-(1-\alpha)} & \text { if } \mu>0 .\end{cases}
$$

If $\mu<0$ the function $\zeta(y)$ is strictly decreasing from $+\infty$ to $-\infty$ on $(0,+\infty)$ and has a zero at $\hat{y}_{1}$, if $\mu=0$ it is strictly decreasing from $+\infty$ to 0 on $(0,+\infty)$, if $\mu>0$ it is strictly decreasing from $+\infty$ to $\hat{x}_{1}$ on $\left(0, \hat{y}_{1}\right]$, and increases again to $+\infty$ on $\left[\hat{y}_{1},+\infty\right)$.

Proof: We have

$$
\zeta^{\prime}(y)=\frac{\mu}{\beta}-\frac{1}{\beta} y^{-\frac{1}{1-\alpha}}, \quad y>0 .
$$

This shows: If $\mu<0$ then $\zeta^{\prime}(y)<0, \zeta(y) \rightarrow-\infty$ as $y \rightarrow+\infty$, and $\zeta\left(\hat{y}_{1}\right)=0$. If $\mu=0$ then $\zeta^{\prime}(y)<0$ and $\zeta(y) \rightarrow 0$ as $y \rightarrow \infty$. If $\mu>0$ we have $\zeta^{\prime}\left(\hat{y}_{1}\right)=0$ and this is the only zero of $\zeta(y)$. Moreover $\hat{x}_{1}=\zeta\left(\hat{y}_{1}\right)$.

Lemma 6 The function $\zeta(y)$ admits an increasing inverse function $\bar{\theta}(x)$ for $x \in\left(\hat{x}_{1},+\infty\right)$ and a decreasing inverse function $\underline{\theta}(x)$ for $x \in\left(\hat{x}_{1},+\infty\right)$. The limiting behavior of $\underline{\theta}(x)$ for $x \rightarrow \infty$ is given by

$$
\underline{\theta}(x)=\left(\frac{1-\alpha}{\alpha \beta}\right)^{\frac{1-\alpha}{\alpha}} x^{-\frac{1-\alpha}{\alpha}}+\mathcal{O}\left(x^{-\frac{2-\alpha}{\alpha}}\right), \quad x \rightarrow+\infty .
$$

Proof: We know from the previous lemma, that the restriction of $\zeta(y)$ to $\left(\hat{y}_{1},+\infty\right)$ is strictly increasing, thus we have the increasing inverse function $\bar{\theta}(x)$. The restriction of $\zeta(y)$ to $\left(0, \hat{y}_{1}\right)$ is strictly decreasing from $+\infty$ to $\hat{x}_{1}$, and thus admits an inverse function $\underline{\theta}(x)$ on $\left(\hat{x}_{1},+\infty\right)$ that decreases from $\hat{y}_{1}$ to 0 . Thus $x \geq \hat{x}_{1}$ implies $\underline{\theta}(x) \leq \hat{y}_{1}$. If $\mu=0$ we have

$$
\underline{\theta}(x)=\left(\frac{1-\alpha}{\alpha \beta}\right)^{\frac{1-\alpha}{\alpha}} x^{-\frac{1-\alpha}{\alpha}},
$$

and (A.8) holds with zero remainder. If $\mu \neq 0$ we can rewrite the equation $x=\zeta(\underline{\theta}(x))$ for sufficiently large $x$ as

$$
\underline{\theta}(x)=\left(\frac{1-\alpha}{\alpha \beta}\right)^{\frac{1-\alpha}{\alpha}} x^{-\frac{1-\alpha}{\alpha}}\left(1-\mu \frac{\underline{\theta}(x)}{x}\right)^{-\frac{1-\alpha}{\alpha}}
$$


A first crude estimate is therefore

$$
\underline{\theta}(x)=\mathcal{O}\left(x^{-\frac{1-\alpha}{\alpha}}\right) .
$$

Expanding the last factor on the right hand side of (A.10) yields

$$
\begin{aligned}
\underline{\theta}(x) & =\left(\frac{1-\alpha}{\alpha \beta}\right)^{\frac{1-\alpha}{\alpha}} x^{-\frac{1-\alpha}{\alpha}}\left(1+\mathcal{O}\left(\frac{\underline{\theta}(x)}{x}\right)\right) \\
& =\left(\frac{1-\alpha}{\alpha \beta}\right)^{\frac{1-\alpha}{\alpha}} x^{-\frac{1-\alpha}{\alpha}}+\mathcal{O}\left(\underline{\theta}(x) x^{-\frac{1}{\alpha}}\right)
\end{aligned}
$$

Inserting the crude estimate (A.11) shows (A.8).

\section{A.2 Inflection points}

Suppose $y(x)$ is a solution of the ODE (A.1). Differentiating the equation $y^{\prime}(x)=F(x, y(x))$ we obtain

$$
\begin{aligned}
y^{\prime \prime}(x) & =F_{x}(x, y(x))+F_{y}(x, y(x)) y^{\prime}(x) \\
& =F_{x}(x, y(x))+F_{y}(x, y(x)) F(x, y(x)) .
\end{aligned}
$$

Hence, if $x$ is an inflection point of the function $y(x)$, then $(x, y(x))$ lies on

$$
F_{x}(x, y)+F_{y}(x, y) F(x, y)=0 .
$$

\section{Lemma 7 Let}

$$
G(x, y)=F_{x}(x, y)+F_{y}(x, y) F(x, y) .
$$

\section{Then we have}

(i) The solutions of $G(x, y)=0$ with $(x, y) \in \mathbb{R} \times \mathbb{R}_{>0}$ can be parameterized by $x=\xi_{1}(y)$ and $x=\xi_{2}(y)$, where $\xi_{1}(y)$ and $\xi_{2}(y)$ are given by

$$
\xi_{1}(y)=\frac{y}{2 \beta}\left(\mu+\frac{2-\alpha}{\alpha} y^{-\frac{1}{1-\alpha}}+\sqrt{2 \beta \sigma^{2}+\left(y^{-\frac{1}{1-\alpha}}-\mu\right)^{2}}\right),
$$

and

$$
\xi_{2}(y)=\frac{y}{2 \beta}\left(\mu+\frac{2-\alpha}{\alpha} y^{-\frac{1}{1-\alpha}}-\sqrt{2 \beta \sigma^{2}+\left(y^{-\frac{1}{1-\alpha}}-\mu\right)^{2}}\right) .
$$


(ii) We have the asymptotic estimates

$$
\xi_{1}(y)= \begin{cases}\frac{1}{\alpha \beta} y^{-\frac{\alpha}{1-\alpha}}+\mathcal{O}\left(y^{\frac{2-\alpha}{1-\alpha}}\right) & y \rightarrow 0 \\ \left(\frac{\mu+\sqrt{\mu^{2}+2 \beta \sigma^{2}}}{2 \beta}\right) y+\mathcal{O}\left(y^{-\frac{\alpha}{1-\alpha}}\right) & y \rightarrow \infty\end{cases}
$$

and

$$
\xi_{2}(y)= \begin{cases}\frac{1-\alpha}{\alpha \beta} y^{-\frac{\alpha}{1-\alpha}}+\frac{\mu}{\beta} y+\mathcal{O}\left(y^{\frac{2-\alpha}{1-\alpha}}\right) & y \rightarrow 0 \\ \left(\frac{\mu-\sqrt{\mu^{2}+2 \beta \sigma^{2}}}{2 \beta}\right) y+\mathcal{O}\left(y^{-\frac{\alpha}{1-\alpha}}\right) y \rightarrow \infty .\end{cases}
$$

(iii) There is $\hat{y}_{2}>0$ such that $\xi_{1}(y)$ is strictly decreasing from $+\infty$ to $\xi_{1}\left(\hat{y}_{2}\right)$ on $\left(0, \hat{y}_{2}\right]$ and then increases again to $+\infty$ on $\left[\hat{y}_{2},+\infty\right)$. The function $\xi_{2}(y)$ is strictly decreasing from $+\infty$ to $-\infty$ on $(0,+\infty)$.

(iv) We have the inequality

$$
0<\xi_{2}(y)<\zeta(y)<\xi_{1}(y), \quad y>0
$$

Proof: (i) We have

$$
G(x, y)=g(y)\left(x^{2}+p(y) x+q(y)\right)
$$

with

$$
p(y)=-\frac{y}{\beta}\left(\mu+\frac{2-\alpha}{\alpha} y^{-\frac{1}{1-\alpha}}\right),
$$

and

$$
g(y)=-\frac{4 \beta^{2}}{\sigma^{4} y^{3}}, \quad q(y)=-\frac{y^{2}}{\beta^{2}}\left(\frac{\beta \sigma^{2}}{2}-\frac{\mu}{\alpha} y^{-\frac{1}{1-\alpha}}-\frac{1-\alpha}{\alpha^{2}} y^{-\frac{2}{1-\alpha}}\right) .
$$

Thus $G(x, y)=0$ is equivalent to a quadratic equation in $x$ with solutions $\xi_{1}(y)=-\frac{1}{2} p(y)+\sqrt{r(y)}$ and $\xi_{2}(y)=-\frac{1}{2} p(y)-\sqrt{r(y)}$, where $r(y)=p(y)^{2} / 4-$ $q(y)$.

(ii) The asymptotic expansions are elementary. Let us consider $y \rightarrow 0$ first. We have

$$
r(y)=\frac{1}{4 \beta^{2}} y^{-\frac{2 \alpha}{1-\alpha}}\left(1-2 \mu y^{\frac{1}{1-\alpha}}+\left(\mu^{2}+2 \beta \sigma^{2}\right) y^{\frac{2}{1-\alpha}}\right),
$$


and expanding the square root gives

$$
\sqrt{r(y)}=\frac{1}{2 \beta} y^{-\frac{\alpha}{1-\alpha}}\left(1-\mu y^{\frac{1}{1-\alpha}}+\mathcal{O}\left(y^{\frac{2}{1-\alpha}}\right)\right) .
$$

Next consider $y \rightarrow \infty$. We have

$$
r(y)=\frac{\mu^{2}+2 \beta \sigma^{2}}{4 \beta^{2}} y^{2}\left(1-\frac{2 \mu}{\mu^{2}+2 \beta \sigma^{2}} y^{-\frac{1}{1-\alpha}}+\frac{1}{\mu^{2}+2 \beta \sigma^{2}} y^{-\frac{2}{1-\alpha}}\right),
$$

and expanding the square root yields

$$
\sqrt{r(y)}=\frac{\sqrt{\mu^{2}+2 \beta \sigma^{2}}}{2 \beta} y\left(1+\mathcal{O}\left(y^{-\frac{1}{1-\alpha}}\right)\right) .
$$

(iii) Next let us look at the derivatives

$$
\xi_{1,2}^{\prime}(y)=-\frac{1}{2} p^{\prime}(y) \pm \frac{r^{\prime}(y)}{2 \sqrt{r(y)}} .
$$

We have $\xi_{1}(y)=0$ resp. $\xi_{2}(y)=0$ if

$$
p^{\prime}(y)= \pm \frac{r^{\prime}(y)}{2 \sqrt{r(y)}}
$$

Squaring yields

$$
r(y) p^{\prime}(y)^{2}-r^{\prime}(y)^{2}=0,
$$

or

$$
\frac{y^{2}}{(1-\alpha) \beta^{4}} A\left(y^{-\frac{1}{1-\alpha}}\right)=0
$$

where

$$
\begin{aligned}
A(z)= & z^{4}-3 \mu z^{3}+\left(3 \mu^{2}+\frac{4-2 \alpha-\alpha^{2}}{2(1-\alpha)} \beta \sigma^{2}\right) z^{2} \\
& -\left(\mu^{2}+(1+\alpha) \beta \sigma^{2}\right) \mu z-(1-\alpha) \beta \sigma^{2}\left(\frac{\mu^{2}}{2}+\beta \sigma^{2}\right) .
\end{aligned}
$$


In Lemma 8 below we show, that this quartic equation has exactly one positive solution, that we denote by $\hat{z}_{2}$. This means that $\hat{y}_{2}=\hat{z}_{2}^{-(1-\alpha)}$ is the only candidate for a zero of $\xi_{1}^{\prime}(y)$ or $\xi_{2}^{\prime}(y)$. We know from the asymptotics that $\xi_{1}(y)$ must have a local minimum, thus $\xi_{1}^{\prime}\left(\hat{y}_{2}\right)=0$. If both $\xi_{1}^{\prime}(y)$ and $\xi_{2}^{\prime}(y)$ vanished at the same $y$, then, by Vièta's formulas, we would have $p^{\prime}(y)$ and $q^{\prime}(y)=0$. As

$$
\begin{aligned}
q^{\prime}(y) & +\frac{y}{\beta}\left(\frac{\mu^{2}}{(2-\alpha)^{2}}+\frac{2(1-\alpha)}{\alpha(2-\alpha)} y^{-\frac{1}{1-\alpha}}\right) p^{\prime}(y) \\
& =-\frac{y}{\beta^{2}}\left(\frac{\mu^{2}}{(2-\alpha)^{2}}+\beta \sigma^{2}\right)
\end{aligned}
$$

we see that $\xi_{1}^{\prime}(y)$ and $\xi_{2}^{\prime}(y)$ never vanish simultaneously for $y>0$. Thus $\xi_{2}^{\prime}(y)$ has no zeroes and, by looking at the asymptotics, we see that $\xi_{2}(y)$ is a strictly decreasing function.

(iv) Looking at the definition of $G(x, y)$ in (A.17) we see that $F(x, y)=0$ and $G(x, y)=0$ do not have a common solution with $y>0$. In other words the set of possible inflection points and the set of possible critical points do not intersect. This fact, combined with the asymptotic expansions, and the smoothness of the functions implies, that $\xi_{1}(y)$ runs to the top resp. right and $\xi_{2}(y)$ to the bottom resp. left of $\zeta(y)$, more precisely, the inequality (A.22).

\section{Lemma 8 Suppose}

$$
\begin{aligned}
A(z)= & z^{4}-3 \mu z^{3}+\left(3 \mu^{2}+\frac{4-2 \alpha-\alpha^{2}}{2(1-\alpha)} \beta \sigma^{2}\right) z^{2} \\
& -\left(\mu^{2}+(1+\alpha) \beta \sigma^{2}\right) \mu z-(1-\alpha) \beta \sigma^{2}\left(\frac{\mu^{2}}{2}+\beta \sigma^{2}\right) .
\end{aligned}
$$

with $\mu \in \mathbb{R}, 0<\alpha<1, \beta>0, \sigma>0$. Then $A(z)=0$ has exactly one positive solution.

Proof: If $\mu=0$ then the equation is biquadratic and the solutions are fairly simple. In the general case we do not want to write down Ferrari's explicit formula. Sturm sequences also yield lengthy expressions. Therfore we investigate the behavior of the equation in the following elementary way. We use

$$
\begin{aligned}
A^{\prime}(z)= & 4 z^{3}-9 \mu z^{2}+\left(6 \mu^{2}+\frac{4-2 \alpha-\alpha^{2}}{1-\alpha} \beta \sigma^{2}\right) z \\
& -\left(\mu^{2}+(1-\alpha) \beta \sigma^{2}\right) \mu-(1-\alpha) \beta \sigma^{2}\left(\frac{\mu^{2}}{2}+\beta \sigma^{2}\right) .
\end{aligned}
$$


and

$$
A^{\prime \prime}(z)=12 z^{2}-18 \mu z+\left(6 \mu^{2}+\frac{4-2 \alpha-\alpha^{2}}{1-\alpha} \beta \sigma^{2}\right)
$$

We observe that

$$
A(0)<0, \quad A^{\prime}(0)<0, \quad A^{\prime \prime}(0)>0 .
$$

Let

$$
\mu_{0}=\sqrt{\frac{4\left(4-2 \alpha-\alpha^{2}\right) \beta \sigma^{2}}{3(1-\alpha)}} .
$$

If $|\mu|<\mu_{0}$ then $A^{\prime \prime}(z)$ has no real zeroes, if $\mu=\mu_{0}$ a double zero, if $|\mu|>\mu_{0}$ two real zeroes. If $|\mu|<\mu_{0}$ this implies, that $A(z)$ has no inflection points, is convex, and since $A(0)<0$, we have exactly one negative and one positive zero of $A(z)$. If $\mu \leq 0$ then $A^{\prime}(z)>0$ for $z>0, A(z)$ is therefore increasing on $z>0$, and since $A(0)<0$ the same conclusion follows. It remains to treat $\mu \geq \mu_{0}$. The two inflection points of $A(z)$ are

$$
\tilde{z}_{1}=\frac{3 \mu-\sqrt{\mu^{2}-\mu_{0}^{2}}}{4}, \quad \tilde{z}_{2}=\frac{3 \mu+\sqrt{\mu^{2}-\mu_{0}^{2}}}{4} .
$$

We have $0<\tilde{z}_{1}<\tilde{z}_{2}$. At $\tilde{z}_{1}$ we have a local maximum of $A^{\prime}(z)$, at $\tilde{z}_{2}$ a local minimum. Moreover

$$
\begin{aligned}
A^{\prime}\left(\tilde{z}_{2}\right)= & \frac{\mu^{2}}{8}\left(\mu-\sqrt{\mu^{2}-\mu_{0}^{2}}\right) \\
& +\left(\frac{8-6 \alpha+\alpha^{2}}{4(1-\alpha)} \mu+\frac{4-2 \alpha-\alpha^{2}}{6(1-\alpha)} \sqrt{\mu^{2}-\mu_{0}^{2}}\right) \beta \sigma^{2}>0 .
\end{aligned}
$$

As $A^{\prime}(0)<0$ this implies that $A^{\prime}(z)$ has exactly one real zero $\bar{z}_{0}$, and that zero satisfies $0<\bar{z}_{0}<\tilde{z}_{1}$. Thus $A(z)$ is decreasing for $z \leq \bar{z}_{0}$ and increasing for $z \geq \bar{z}_{0}$. Hence $A(z)$ has exactly one positive zero $z_{0}$, and that zero satisfies $z_{0}>\bar{z}_{0}$.

Lemma 9 The function $\xi_{1}(y)$ admits an inverse function $\bar{\eta}_{1}(x)$, that increases from $\hat{y}_{2}$ at $\hat{x}_{2}$ to $+\infty$ as $x \rightarrow+\infty$, and inverse function $\underline{\eta}_{2}(x)$, that decreases from $\hat{y}_{2}$ at $\hat{x}_{2}$ to 0 as $x \rightarrow+\infty$, where $\hat{x}_{2}=\xi_{1}\left(\hat{y}_{2}\right)$. The function $\xi_{2}(y)$ admits a decreasing inverse function $\eta_{2}(x)$ that goes to $+\infty$ as $x \rightarrow-\infty$ and to 0 as $x \rightarrow+\infty$. We have

$$
0<\eta_{2}(x)<\underline{\theta}(x)<\underline{\eta}_{1}(x)<\bar{\eta}_{1}(x)<\bar{\theta}(x), \quad\left(x>\hat{x}_{2}\right) .
$$


We have the asymptotics

$$
\underline{\eta}_{1}(x)=\frac{1}{(\alpha \beta)^{\frac{1-\alpha}{\alpha}}} x^{-\frac{1-\alpha}{\alpha}}+\mathcal{O}\left(x^{-\frac{3-\alpha}{\alpha}}\right), \quad x \rightarrow \infty
$$

and

$$
\eta_{2}(x)=\left(\frac{1-\alpha}{\alpha \beta}\right)^{\frac{1-\alpha}{\alpha}} x^{-\frac{1-\alpha}{\alpha}}+\mathcal{O}\left(x^{-\frac{2-\alpha}{\alpha}}\right), \quad x \rightarrow \infty
$$

Proof: From Lemma 7 we know, that $\xi_{1}(y)$ and $\xi_{2}(y)$ admit decreasing inverse functions $\eta_{1}(x)$ and $\eta_{2}(x)$ on $\left(\hat{x}_{2},+\infty\right)$ resp. on $(0,+\infty)$, that satisfy (A.44). We also know $\eta_{1}(x) \rightarrow 0$ as $x \rightarrow \infty$. Thus we obtain from $x=\xi_{1}\left(\eta_{1}(x)\right)$ and the $y \rightarrow 0$ asymptotics of $\xi_{1}(y)$ the equation

$$
x=\frac{1}{\alpha \beta} \eta_{1}(x)^{-\frac{\alpha}{1-\alpha}}\left(1+\mathcal{O}\left(\eta_{1}(x)^{\frac{2}{1-\alpha}}\right)\right) .
$$

Solving for $\eta_{1}(x)$ and expanding yields

$$
\begin{aligned}
\eta_{1}(x) & =(\alpha \beta)^{-\frac{1-\alpha}{\alpha}} x^{-\frac{1-\alpha}{\alpha}}\left(1+\mathcal{O}\left(\eta_{1}(x)^{\frac{2}{1-\alpha}}\right)\right)^{\frac{1-\alpha}{\alpha}} \\
& =(\alpha \beta)^{-\frac{1-\alpha}{\alpha}} x^{-\frac{1-\alpha}{\alpha}}\left(1+\mathcal{O}\left(\eta_{1}(x)^{\frac{2}{1-\alpha}}\right)\right) .
\end{aligned}
$$

This gives the first crude estimate

$$
\eta_{1}(x)=\mathcal{O}\left(x^{-\frac{1-\alpha}{\alpha}}\right) .
$$

Plugging this estimate into (A.48) yields (A.45). A similar calculations yields (A.46). We already know $\eta_{2}(x) \rightarrow 0$ as $x \rightarrow \infty$. Thus we obtain from $x=$ $\xi_{2}\left(\eta_{2}(x)\right)$ and the $y \rightarrow 0$ asymptotics of $\xi_{2}(y)$ the equation

$$
x=\frac{1-\alpha}{\alpha \beta} \eta_{2}(x)^{-\frac{\alpha}{1-\alpha}}\left(1+\mathcal{O}\left(\eta_{2}(x)^{\frac{1}{1-\alpha}}\right)\right) .
$$

Solving for $\eta_{2}(x)$ and expanding yields

$$
\begin{aligned}
\eta_{2}(x) & =\left(\frac{\alpha \beta}{1-\alpha}\right)^{-\frac{1-\alpha}{\alpha}} x^{-\frac{1-\alpha}{\alpha}}\left(1+\mathcal{O}\left(\eta_{2}(x)^{\frac{1}{1-\alpha}}\right)\right)^{\frac{1-\alpha}{\alpha}} \\
& =\left(\frac{\alpha \beta}{1-\alpha}\right)^{-\frac{1-\alpha}{\alpha}} x^{-\frac{1-\alpha}{\alpha}}\left(1+\mathcal{O}\left(\eta_{2}(x)^{\frac{1}{1-\alpha}}\right)\right) .
\end{aligned}
$$


This gives the first crude estimate

$$
\eta_{2}(x)=\mathcal{O}\left(x^{-\frac{1-\alpha}{\alpha}}\right) .
$$

Plugging this estimate into (A.52) yields (A.46).

Lemma 10 We have the inequality

$$
F\left(x, \eta_{2}(x)\right)<\eta_{2}^{\prime}(x)<0, \quad(x>0) .
$$

Proof: We recall, that

$$
G(x, y)=g(y)\left(x^{2}+p(y) x+q(y)\right) .
$$

Thus

$$
G_{x}(x, y)=g(y)(2 x+p(y))
$$

Now let us consider a point $\left(x_{0}, y_{0}\right)$ such that $y_{0}=\eta_{2}\left(x_{0}\right)$, or equivalently, that $x_{0}=\xi_{2}\left(y_{0}\right)$. Using $\xi_{2}\left(y_{0}\right)=-p\left(y_{0}\right) / 2-\sqrt{r\left(y_{0}\right)}$ yields

$$
G_{x}\left(x_{0}, y_{0}\right)=-2 g\left(y_{0}\right) \sqrt{r\left(y_{0}\right)}<0 .
$$

Differentiating $G\left(x, \eta_{2}(x)\right)=0$ at $x=x_{0}$ gives

$$
G_{x}\left(x_{0}, y_{0}\right)+G_{y}\left(x_{0}, y_{0}\right) \eta_{2}^{\prime}\left(x_{0}\right)=0 \text {. }
$$

We know already, that $\eta_{2}(x)$ is strictly decreasing, so $\eta_{2}^{\prime}\left(x_{0}\right)<0$, thus we must have $G_{y}\left(x_{0}, y_{0}\right)>0$. We can write

$$
\eta_{2}^{\prime}\left(x_{0}\right)=-\frac{G_{x}\left(x_{0}, y_{0}\right)}{G_{y}\left(x_{0}, y_{0}\right)}
$$

Our claim is equivalent to

$$
F\left(x_{0}, y_{0}\right)<-\frac{G_{x}\left(x_{0}, y_{0}\right)}{G_{y}\left(x_{0}, y_{0}\right)}
$$

or $H\left(x_{0}, y_{0}\right)<0$ with

$$
H(x, y)=G_{x}(x, y)+G_{y}(x, y) F(x, y) .
$$


Observing $x_{0}=\xi_{2}\left(y_{0}\right)$ gives

$$
H\left(x_{0}, y_{0}\right)=\ell\left(y_{0}\right) \sqrt{r\left(y_{0}\right)}+m\left(y_{0}\right)
$$

where

$$
\ell(y)=\frac{8 \beta}{(1-\alpha) \sigma^{6}} y^{-\frac{4-3 \alpha}{1-\alpha}}\left(y^{-\frac{1}{1-\alpha}}-\mu\right)
$$

and

$$
m(y)=-\frac{4}{(1-\alpha) \sigma^{6}} y^{-\frac{3-2 \alpha}{1-\alpha}}\left(\left(y^{-\frac{1}{1-\alpha}}-\mu\right)^{2}+\beta \sigma^{2}\right) .
$$

We note, that $m(y)<0$ for all $y>0$. If $\ell\left(y_{0}\right)<0$ we are done, if $\ell\left(y_{0}\right) \geq 0$ our claim is equivalent to $\ell\left(y_{0}\right) \sqrt{r\left(y_{0}\right)}<-m\left(y_{0}\right)$, with both sides now nonnegative. We can square and have to show $\ell\left(y_{0}\right)^{2} r\left(y_{0}\right)-m\left(y_{0}\right)^{2}<0$. But

$$
\ell\left(y_{0}\right)^{2} r\left(y_{0}\right)-m\left(y_{0}\right)^{2}=-\frac{16 \beta^{2}}{(1-\alpha)^{2} \sigma^{8}} y^{-\frac{6-4 \alpha}{1-\alpha}} .
$$

\section{A.3 Upper solutions}

Lemma 11 For any $b>\hat{x}_{1}$ there exists $\varepsilon>0$ such that

$$
y^{\prime}=F(x, y), \quad y(b)=\theta(b)
$$

has a unique analytic solution in $(b-\varepsilon, b+\varepsilon)$ satisfying

$$
y^{\prime}(b)=0, \quad y^{\prime \prime}(b)>0
$$

and the estimates

$$
\theta(b)<y(x)<\theta(x) \quad x-\varepsilon<x<b
$$

and

$$
y(x)>\theta(b) \quad b<x<b+\varepsilon .
$$


Proof: The local existence and uniqueness is standard, see, e.g., (Birkhoff and Rota, 1969, Theorem 6.10 .9$, p.171). Since $\theta(x)$ was defined by $F(x, \theta(x))=0$ we have $y^{\prime}(b)=0$ and from

$$
y^{\prime \prime}(x)=F_{x}(x, y(x))+F_{y}(x, y(x)) y^{\prime}(x)
$$

and the initial condition we get

$$
y^{\prime \prime}(b)=\frac{2 \beta}{\sigma^{2} b}>0
$$

We will denote the solution from the previous lemma by $\bar{y}_{b}(x)$.

Lemma 12 Each $\bar{y}_{b}(x)$ can be continued to a solution for all $x \in \mathbb{R}_{>0}$, that satisfies

$$
\theta(b)<\bar{y}_{b}(x)<\theta(x), \quad\left(\hat{x}_{1}<x<b\right) .
$$

Proof: Let us denote by $\left(x_{L}, x_{R}\right)$ the maximal interval, where the solution $\bar{y}_{b}(x)$ can be continued. First we show $\bar{y}_{b}(x)<\theta(x)$ for $x_{L} \wedge \hat{x}_{1}<x<b$ : The following picture, that visualizes the properties from the previous lemma, should suffice, but we will give the details below.

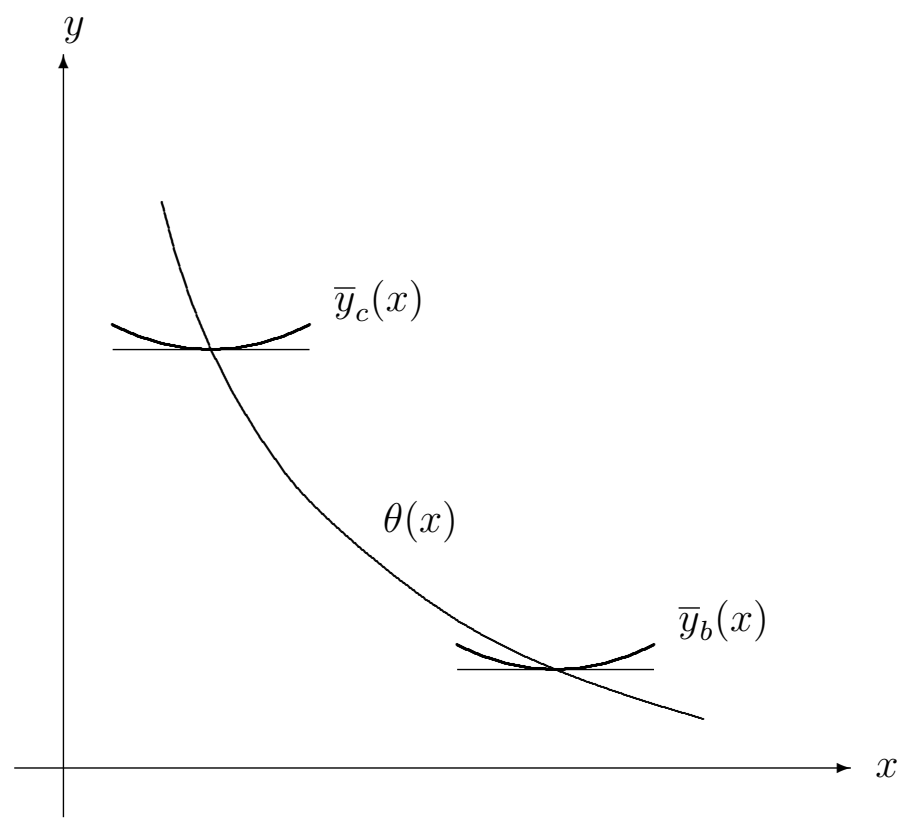

Assume, by contradiction, that $y_{b}(x)=\theta(x)$ for some $x \in\left(x_{L} \wedge \hat{x}_{1}, b\right)$. Let $c=\sup \left\{x \in\left(x_{L} \wedge \hat{x}_{1}, b\right): \bar{y}_{b}(x) \geq \eta(x)\right\}$. By Lemma 11 we have $c<b$. As $\bar{y}_{b}(x)$ is continuous at $x=c$ and we must have $\bar{y}_{b}(c)=0$. By local uniqueness 
we have $\bar{y}_{b}(x)=\bar{y}_{c}(x)$ in a neighborhood of $x=c$. But the previous lemma tells us that $\bar{y}_{c}(\tilde{c})>\theta(\tilde{c})$ for some $\tilde{c} \in(c, b)$, contradicting the definition of $c$.

Now we know $\bar{y}_{b}(x)<\theta(x)$ for $x_{L} \wedge \hat{x}_{1}<x<b$. This implies $\bar{y}_{b}^{\prime}(x)<0$ for $x_{L}<x<b$ and thus $\bar{y}_{b}(x) \geq \theta(b)$ in $x_{L}<x \leq b$. This means, the solution $\bar{y}_{b}(x)$ stays away from the singularities of $F(x, y)$. On the other hand $F(x, y)$ is (at most) linearly bounded in both variables on $(-\infty, b] \times[\theta(b),+\infty)$, and a crude estimate implies, that the solution does not explode to infinity for $x_{L}<x<b$. As a consequence of a standard theorem, e.g, (Birkhoff and Rota, 1969, 6.11.10, p.172) (with left and right boundary interchanged), we must have $x_{L}=-\infty$.

A similar argument shows that $\bar{y}_{b}(x)>\theta(x)$ for $b<x<X_{R}$. Thus $\bar{y}_{b}^{\prime}(x)>0$ for $x>b$ and therefore $\bar{y}_{b}(x)>\theta(b)$ in that interval. Precisely the same argument as before shows $x_{R}=+\infty$.

\section{A.4 Lower solutions}

Lemma 13 For any $b>0$ there exists $\varepsilon>0$ such that

$$
y^{\prime}=F(x, y), \quad y(b)=\eta_{2}(b)
$$

has a unique analytic solution in $(b-\varepsilon, b+\varepsilon)$ satisfying

$$
y^{\prime}(b)<\eta_{2}^{\prime}(b), \quad y^{\prime \prime}(b)=0
$$

and the estimates

$$
\eta_{2}(x)<y(x), \quad x-\varepsilon<x<b
$$

and

$$
0<y(x)<\eta_{2}(x) \quad b<x<b+\varepsilon .
$$

Proof: Local existence and uniqueness is again standard as above. From the prescribed condition $y(b)=\eta_{2}(b)$ we get $y^{\prime \prime}(b)=G(b, y(b))=0$. From Lemma 10 we see $y^{\prime}(b)=F(b, y(b))<\eta_{2}^{\prime}(b)$, meaning, that the tangent of $y(x)$ is steeper than the tangent of $\eta_{2}(x)$ in $x=b$. Taking $\epsilon>0$ sufficiently small yields the inequalities (A.76) and (A.77).

We will denote the solution from the previous lemma by $\underline{y}_{b}(x)$. 
Lemma 14 For each $b>0$ exists a finite $x_{R}(b)>b$ such that $\underline{y}_{b}(x)$ can be continued to a solution on $\left(-\infty, x_{R}(b)\right)$, that satisfies

$$
\eta_{2}(x)<\underline{y}_{b}(x)<\bar{y}_{b}(x), \quad x \in(-\infty, b),
$$

and

$$
0<\underline{y}_{b}(x)<\eta_{2}(x), \quad x \in\left(b, x_{R}(b)\right)
$$

and we have

$$
\lim _{x \rightarrow x_{R}(b)} \underline{y}_{b}(x)=0, \quad \lim _{x \rightarrow x_{R}(b)} \underline{y}_{b}^{\prime}(x)=-\infty .
$$

Proof: Let us denote by $\left(x_{L}, x_{R}\right)$ the maximal interval, where the solution $\underline{y}_{b}(x)$ can be continued. First we show $\underline{y}_{b}(x)>\eta_{2}(x)$ for $x \in\left(x_{L}, b\right)$. The following picture, that visualizes the properties from the previous lemma, should suffice, but, again, we give the details below.

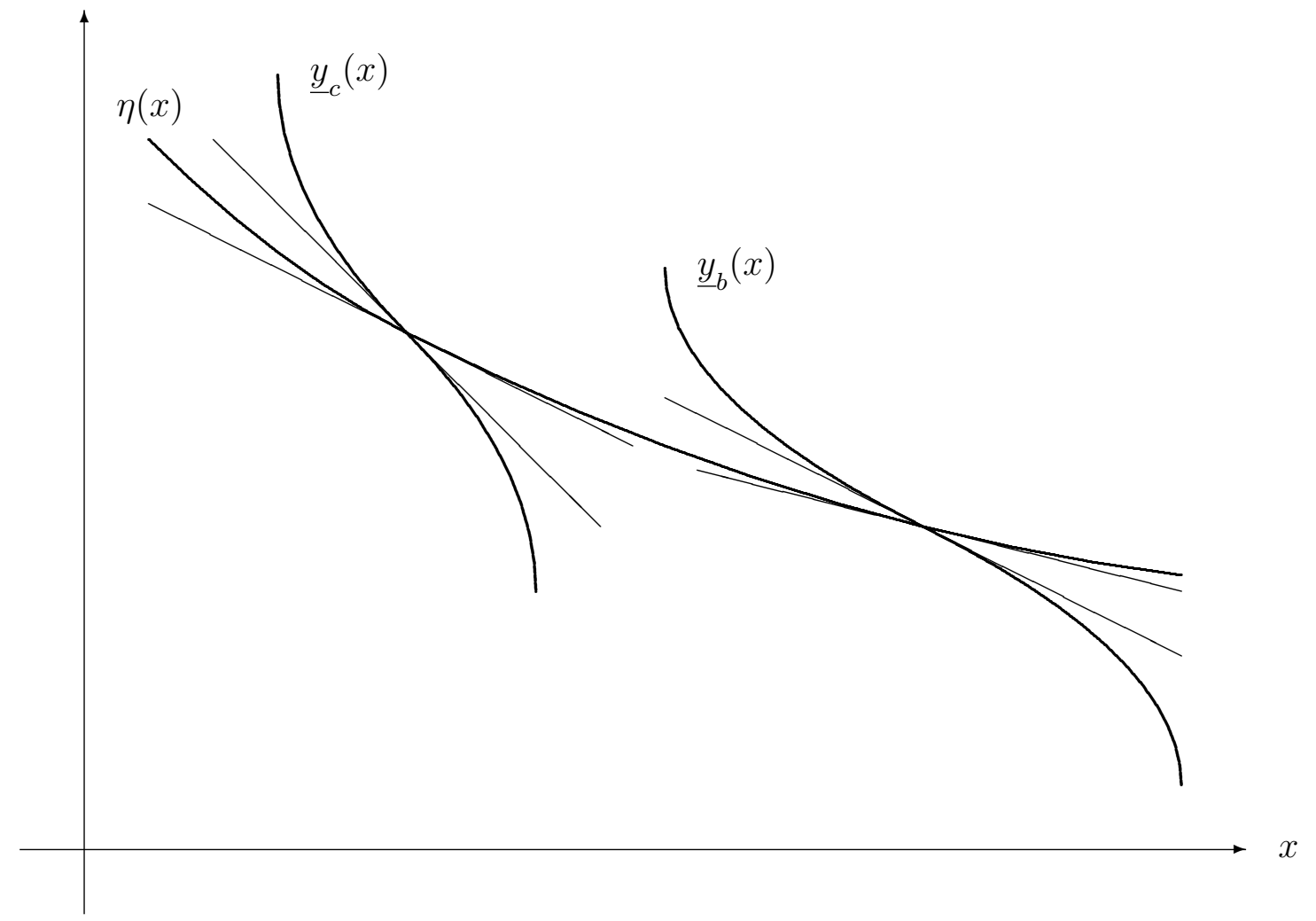

Assume by contradiction, that $y_{b}(x)=\eta(x)$ for some $x \in\left(x_{L}, b\right)$. Let $c=$ $\sup \left\{x \in\left(x_{L}, b\right): \underline{y}_{b}(x) \leq \eta(x)\right\}$. By Lemma 13 we have $c<b$, from the continuity of $\underline{y}_{b}(x)$ we get $\underline{y}_{b}(c) \leq \eta(c)$. By local uniqueness we have $\underline{y}_{b}(x)=$ 
$\underline{y}_{c}(x)$ around $x=c$. But Lemma 13 tells us, that $\underline{y}_{c}(\tilde{c})<\eta(\tilde{c})$ for some $\tilde{c} \in(c, b)$ contradicting the definition of $c$.

Now we know $\underline{y}_{b}(x)>\eta(x)$ on $\left(x_{L}, b\right)$ and thus $\underline{y}_{b}(x)$ is bounded away from the singularities of $F(x, y)$ there. As $F(x, y)$ is (at most) linearly bounded on $(-\infty, b] \times[\eta(b),+\infty)$ the function $\underline{y}_{b}(x)$ cannot explode to $+\infty$. Thus we must have $x_{L}=-\infty$.

A similar argument shows $\underline{y}_{b}(x)<\eta(x)$ for $x \in\left(b, x_{R}\right)$. Assume by contradiction, that $\underline{y}_{b}(x)=\eta(x)$ for some $x \in\left(b, x_{R}\right)$. Let $c=\inf \left\{x \in\left(b, x_{R}\right)\right.$ : $\left.\underline{y}_{b}(x) \geq \eta(x)\right\}$. By Lemma 13 we have $c>b$, from the continuity of $\underline{y}_{b}(x)$ we get $y_{b}(c) \geq \eta(c)$. By local uniqueness we have $y_{b}(x)=y_{c}(x)$ around $x=c$. But Lemma 13 tells us, that $\underline{y}_{c}(\tilde{c})>\eta(\tilde{c})$ for some $\tilde{c} \in(b, c)$ contradicting the definition of $c$.

Now we know $\underline{y}_{b}(x)<\eta(x)$. This means, that $\underline{y}_{b}(x)$ is decreasing and concave on $\left(b, x_{R}\right)$. Thus $x_{R}=+\infty$ is impossible as $\underline{y}_{b}(x)$ must eventually hit zero. On the other hand we must have $\lim _{x \uparrow x_{R}} \underline{y}(x)=0$, because otherwise $\lim _{x \uparrow x_{R}} \underline{y}(x)$ would exist, were greater than zero, and we could continue $\underline{y}_{b}(x)$ further to the right, contradicting the definition of $x_{R}$.

\section{A.5 The critical solution}

Lemma 15 There is a unique decreasing positive solution $y_{\infty}(x)$ of the $O D E$ on $\mathbb{R}$, that satisfies

$$
y_{\infty}(x)=\lim _{b \rightarrow \infty} \underline{y}_{b}(x)=\lim _{b \rightarrow \infty} \bar{y}_{b}(x)
$$

and

$$
\eta(x)<y_{\infty}(x), \quad x \in \mathbb{R}
$$

and

$$
y_{\infty}(x)<\theta(x), \quad x>\hat{x}_{1} .
$$

Consequently $y_{\infty}(x)$ is convex and satisfies

$$
y_{\infty}(x)=\left(\frac{1-\alpha}{\alpha \beta}\right)^{\frac{1-\alpha}{\alpha}} x^{-\frac{1-\alpha}{\alpha}}+\mathcal{O}\left(x^{-\frac{3-\alpha}{\alpha}}\right), \quad x \rightarrow \infty .
$$




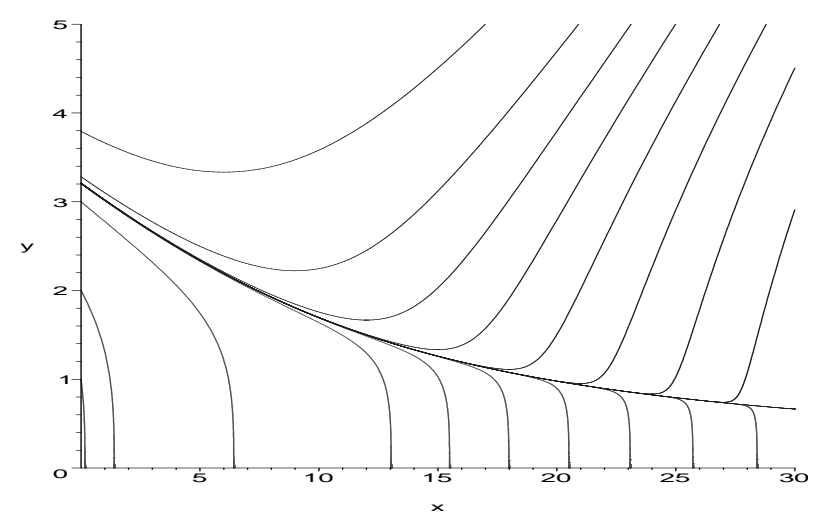

Fig. A.1. Solutions $y(x)$ of the ODE with $\mu=0, \sigma=1, \alpha=0.5, \beta=0.05$

Proof: The solutions $\bar{y}_{b}(x)$ do not intersect each other. Thus $\bar{y}_{b}(x)$ is decreasing in $b>\hat{x}_{1}$ for all $x \in \mathbb{R}$. They are bounded below by $\bar{y}_{b}(x)>\eta(x)>0$. Thus we can define $\bar{y}_{\infty}(x)=\lim _{b \rightarrow \infty} \bar{y}_{b}(x)$. Let us fix two real numbers $\ell<r$ and $\epsilon>0$. The functions $\bar{y}_{b}(x)$ with $b>b_{0}=(r+\epsilon) \vee \hat{x}_{1}$ are convex and decreasing on $(\ell-\epsilon, r+\epsilon)$, thus the limit $\bar{y}_{\infty}(x)$ is also convex and decreasing on that interval. In particular $\bar{y}_{\infty}(x)$ is continuous on $[\ell, r]$. We have a decreasing sequence of continuous functions converging to a continuous function on a compact interval. By Dini's Theorem we have uniform convergence. We have $\eta(r) \leq \bar{y}_{b}(x) \leq \theta(\ell)$ for $x \in[\ell, r]$ and $b \geq b_{0}$, hence $F\left(x, \bar{y}_{b}(x)\right)$ is also uniformly convergent on $[\ell, r]$. To summarize, we have uniform convergence $\bar{y}_{b}(x) \rightarrow \bar{y}_{\infty}(x)$ and $\bar{y}_{b}^{\prime}(x) \rightarrow F\left(x, \bar{y}_{b}(x)\right)$ on $[\ell, r]$. This is more than we need to show that $\bar{y}_{\infty}(x)$ is differentiable and satisfies the ODE.

A similar argument as above, now with $\underline{y}_{b}(x)$ increasing in $b$ for all $x$, shows that $\underline{y}_{\infty}(x)=\lim _{b \rightarrow \infty} \underline{y}_{b}(x)$ exists, is convex, decreasing, differentiable, and satisfies the ODE.

To show that $\bar{y}_{\infty}(x)=\underline{y}_{\infty}(x)$ we observe, that

$$
\underline{y}_{\infty}(x) \leq \bar{y}_{\infty}(x)<\theta(x)<(\alpha \beta x)^{-\frac{1-\alpha}{\alpha}}
$$

for sufficiently large $x$. This implies that $\left(x, \underline{y}_{\infty}(x)\right)$ and $\left(x, \bar{y}_{\infty}(x)\right)$ both lie in the area where $F_{y}(x, y) \geq 0$ and thus

$$
\underline{y}_{\infty}^{\prime}(x)=F\left(x, \underline{y}_{\infty}(x)\right) \leq F\left(x, \bar{y}_{\infty}(x)\right)=\bar{y}_{\infty}^{\prime}(x) .
$$

As a consequence, if we had $\underline{y}_{\infty}(c)=\bar{y}_{\infty}(c)-\epsilon$ for some $c$ sufficiently large and $\epsilon>0$, then $\underline{y}_{\infty}(x) \leq \bar{y}_{\infty}(x)-\epsilon$ for all $x \geq c$, and eventually $\underline{y}(x)$ had to hit zero, which is not the case. 
A.6 Asymptotics for $x \rightarrow \infty$

Proposition 2 Let $v$ be as in Proposition 1. Then (17), (18), and (19) hold as $x \rightarrow \infty$.

Proof: We know from (A.84) in Lemma 15 that

$$
y(v) \sim c v^{-\frac{1-\alpha}{\alpha}}, \quad v \rightarrow \infty .
$$

Fix $x_{0}>0$. We can find constants $c_{1} \in(0, c)$ and $c_{2} \in(c, \infty)$ such that

$$
c_{1} v^{-\frac{1-\alpha}{\alpha}} \leq y(v) \leq c_{2} v^{-\frac{1-\alpha}{\alpha}}, \quad v \geq v\left(x_{0}\right) .
$$

Then we look at the initial value problem

$$
v_{1}^{\prime}=c_{1} v_{1}^{-\frac{1-\alpha}{\alpha}}, \quad v_{1}\left(x_{0}\right)=v\left(x_{0}\right)
$$

This has the solution

$$
v_{1}(x)=\left(\frac{c_{1}}{\alpha}\left(x-x_{0}\right)+v\left(x_{0}\right)^{\frac{1}{\alpha}}\right)^{\alpha}
$$

A standard comparison theorem shows now $v_{1}(x) \leq v(x)$ for $x \geq x_{0}$. A similar results follows for the upper bound and we get

$$
v(x) \asymp x^{\alpha}, \quad x \rightarrow \infty .
$$

Naturally we expect

$$
v(x) \sim\left(\frac{c}{\alpha}\right)^{\alpha} x^{\alpha}, \quad x \rightarrow \infty .
$$

To show that, we use

$$
y(v)=c v^{-\frac{1-\alpha}{\alpha}}+\mathcal{O}\left(v^{-\frac{2-\alpha}{\alpha}}\right), \quad v \rightarrow \infty .
$$

This means, we can find $v_{0}>0$ and $K>0$, such that

$$
c v^{-\frac{1-\alpha}{\alpha}}-K v^{-\frac{2-\alpha}{\alpha}} \leq y(v) \leq c v^{-\frac{1-\alpha}{\alpha}}+K v^{-\frac{2-\alpha}{\alpha}}, \quad v \geq v_{0} .
$$


Then we take $v_{1}=1+\max \left(v_{0},(K / c)^{\alpha}\right)$ to make the left hand side positive for $v \geq v_{1}$. Due to (A.91) we can find $x_{0}>0$, such that $v\left(x_{0}\right) \geq v_{1}$ and consider

$$
\bar{v}^{\prime}=c \bar{v}^{-\frac{1-\alpha}{\alpha}}+K \bar{v}^{-\frac{2-\alpha}{\alpha}}, \quad \bar{v}\left(x_{0}\right)=v\left(x_{0}\right) .
$$

This is (a simple case of) an ODE studied by Conte (1932). It can be solved quite explicitly. Setting

$$
\bar{v}(x)=\left(\frac{c}{\alpha}\right)^{\alpha} z(x)^{-\alpha}, \quad z_{0}=\frac{c}{\alpha} v\left(x_{0}\right)^{-\frac{1}{\alpha}}, \quad k=\frac{\alpha}{c^{2}} K,
$$

we get

$$
z^{\prime}+z^{2}+k z^{3}=0, \quad z\left(x_{0}\right)=z_{0} .
$$

The solution of this equation can be expressed in terms of Lambert's $W$ function, see Corless et al. (1996). This is the inverse of $x e^{x}$. Here we do not need the "main" branch $W(x)$ that increases from the value -1 at $-e^{-1}$ to $+\infty$ as $x \rightarrow \infty$, but another branch, denoted by $W_{-1}(x)$ that decreases from the value -1 at $-e^{-1}$ to $-\infty$ as $x \rightarrow 0$ from the left. We have

$$
z(x)=-\frac{1}{k\left(1+W_{-1}\left(w_{0} e^{w_{0}-\left(x-x_{0}\right) / k}\right)\right)}, \quad w_{0}=-\left(1+\frac{1}{k y_{0}}\right)
$$

Using the asymptotic estimate

$$
W_{-1}(x)=\ln (-x)+\mathcal{O}(\ln (-\ln (-x))), \quad x \rightarrow 0
$$

we obtain, by substitution back into $\bar{v}(x)$,

$$
\bar{v}(x)=\left(\frac{c}{\alpha}\right)^{\alpha} x^{\alpha}\left(1+\mathcal{O}\left(\frac{\ln x}{x}\right)\right), \quad x \rightarrow \infty .
$$

For the lower bound we get a similar estimate. We consider now the equation

$$
\underline{v}^{\prime}=c \underline{v}^{-\frac{1-\alpha}{\alpha}}-K \underline{v}^{-\frac{2-\alpha}{\alpha}}, \quad \underline{v}\left(x_{1}\right)=v\left(x_{1}\right) .
$$

The solution of this equation is

$$
\underline{v}(x)=\left(\frac{c}{\alpha}\right)^{\alpha} y(x)^{-\alpha},
$$


with now

$$
z(x)=\frac{1}{k\left(1+W_{1}\left(w_{0} e^{w_{0}+\left(x-x_{0}\right) / k}\right)\right)} . \quad w_{0}=-\left(1-\frac{1}{k y_{0}}\right) .
$$

Here we need the "main" branch $W_{1}(x)$, often denoted just by $W(x)$, that increases from the value -1 at $-e^{-1}$ to $+\infty$ as $x \rightarrow \infty$. Using the asymptotic estimate

$$
W_{1}(x)=\ln (x)+\mathcal{O}(\ln (\ln (x))), \quad x \rightarrow \infty
$$

we obtain, by substitution back into $\underline{v}(x)$,

$$
\underline{v}(x)=\left(\frac{c}{\alpha}\right)^{\alpha} x^{\alpha}\left(1+\mathcal{O}\left(\frac{\ln x}{x}\right)\right), \quad x \rightarrow \infty .
$$

In general, one cannot simply differentiate an asymptotic expansion. But we can use the differential equation $v_{x}(x)=y(v(x))$ and plug the asymptotics of $v(x)$ into the expansion of $y(v)$ to derive the asymptotics of $v_{x}(x)$ and $c(x)$.

\section{B Convergence details}

As the case $\alpha=1$ only makes sense for positive drift, we assume throughout this section

$$
\mu>0
$$

We emphasize the dependence on $\alpha \in(0,1]$ by writing $\theta^{(\alpha)}(x)$ instead of $\theta(x)$ etc. in the statement of the results, but, for brevity, only occasionally in the proofs.

\section{B.1 Results for the auxiliary functions related to critical points}

Lemma 16 We have the inequalities

$$
\theta^{(\alpha)}(x) \leq \gamma_{\alpha}\left(x-\frac{\mu^{\alpha}}{\beta}\right)^{-\frac{1-\alpha}{\alpha}}, \quad\left(x \geq \frac{\mu^{\alpha}}{\alpha \beta}\right)
$$


and

$$
\theta^{(\alpha)}(x) \geq \gamma_{\alpha} x^{-\frac{1-\alpha}{\alpha}}, \quad\left(x \geq \frac{\mu^{\alpha}}{\alpha \beta}\right),
$$

where

$$
\gamma_{\alpha}=\left(\frac{1-\alpha}{\alpha \beta}\right)^{\frac{1-\alpha}{\alpha}} .
$$

Proof: We recall

$$
\hat{x}_{1}=\frac{\mu^{\alpha}}{\alpha \beta}, \quad \hat{y}_{1}=\mu^{-(1-\alpha)},
$$

and that $\theta$ decreases from $\hat{y}_{1}$ to 0 on $\left[\hat{x}_{1},+\infty\right)$. Thus $x \geq \hat{x}_{1}$ implies $\theta(x) \leq \hat{y}_{1}$. We have

$$
\zeta(y) \leq \frac{\mu}{\beta} \hat{y}_{1}+\frac{1-\alpha}{\alpha \beta} y^{-\frac{\alpha}{1-\alpha}}, \quad\left(0<y \leq \hat{y}_{1}\right) .
$$

Taking $y=\theta(x)$ yields

$$
x=\zeta(\theta(x)) \leq \frac{\mu}{\beta} \hat{y}_{1}+\frac{1-\alpha}{\alpha \beta} \theta(x)^{-\frac{\alpha}{1-\alpha}}, \quad\left(x \geq \hat{x}_{1}\right),
$$

or

$$
x-\frac{\mu}{\beta} \hat{y}_{1} \leq \frac{1-\alpha}{\alpha \beta} \theta(x)^{-\frac{\alpha}{1-\alpha}}, \quad\left(x \geq \hat{x}_{1}\right),
$$

which is equivalent to (B.2). On the other hand we have

$$
\zeta(y) \geq \frac{1-\alpha}{\alpha \beta} y^{-\frac{\alpha}{1-\alpha}}, \quad\left(0<y \leq \hat{y}_{1}\right) .
$$

Taking $y=\theta(x)$ yields

$$
x=\zeta(\theta(x)) \geq \frac{1-\alpha}{\alpha \beta} \theta(x)^{-\frac{\alpha}{1-\alpha}}, \quad\left(x \geq \hat{x}_{1}\right),
$$

which is equivalent to (B.3). 
Lemma 17 We have

$$
\lim _{\alpha \rightarrow 1} \theta^{(\alpha)}(x)=1, \quad\left(x>\frac{\mu}{\beta}\right)
$$

and

$$
\lim _{\alpha \rightarrow 1} \frac{1-\alpha}{\alpha}\left(\theta^{(\alpha)}(x)\right)^{-\frac{\alpha}{1-\alpha}}=\beta x-\mu, \quad\left(x>\frac{\mu}{\beta}\right) .
$$

Proof: We consider a fixed $x>\mu / \beta$. As

$$
\lim _{\alpha \rightarrow 1} \frac{\mu^{\alpha}}{\alpha \beta}=\frac{\mu}{\beta}
$$

we can find $\alpha_{0} \in(0,1)$ such that

$$
x \geq \frac{\mu^{\alpha}}{\alpha \beta}
$$

for $\alpha \in\left(\alpha_{0}, 1\right)$. We obtain from Lemma 16

$$
\gamma_{\alpha} x^{-\frac{1-\alpha}{\alpha}} \leq \theta(x) \leq \gamma_{\alpha}\left(x-\frac{\mu^{\alpha}}{\beta}\right)^{-\frac{1-\alpha}{\alpha}}
$$

Letting $\alpha \rightarrow 1$ shows (B.11). Next we have

$$
x=\frac{\mu}{\beta} \theta(x)+\frac{1-\alpha}{\alpha \beta} \theta(x)^{-\frac{\alpha}{1-\alpha}}
$$

Letting $\alpha \rightarrow 1$ shows (B.12).

B.2 Results for the auxiliary functions related to inflection points

Lemma 18 We have the inequality

$$
\xi_{2}^{(\alpha)}(y) \geq\left(\frac{1-\alpha}{\alpha \beta}\right) y^{-\frac{\alpha}{1-\alpha}}, \quad\left(0<y \leq\left(\frac{2 \mu}{\mu^{2}+2 \beta \sigma^{2}}\right)^{1-\alpha}\right)
$$


Proof: We recall that

$$
\xi_{2}(y)=-\frac{1}{2} p(y)-\sqrt{r(y)},
$$

where

$$
p(y)=-\frac{y}{\beta}\left(\mu+\frac{2-\alpha}{\alpha} y^{-\frac{1}{1-\alpha}}\right),
$$

and

$$
r(y)=\frac{y^{2}}{4 \beta^{2}}\left(2 \beta \sigma^{2}+\left(\mu-y^{-\frac{1}{1-\alpha}}\right)^{2}\right) .
$$

Thus

$$
-\frac{1}{2} p(y) \geq \frac{2-\alpha}{2 \alpha \beta} y^{-\frac{\alpha}{1-\alpha}} .
$$

Next we have

$$
2 \beta \sigma^{2}+\left(\mu-y^{-\frac{1}{1-\alpha}}\right)^{2} \leq y^{-\frac{2}{1-\alpha}},
$$

provided

$$
\left(\mu^{2}+2 \beta \sigma^{2}\right)-2 \mu y^{-\frac{1}{1-\alpha}} \leq 0,
$$

which is equivalent to

$$
y \leq\left(\frac{2 \mu}{\mu^{2}+2 \beta \sigma^{2}}\right)^{1-\alpha} .
$$

Thus

$$
-\sqrt{r(y)} \geq-\frac{1}{2 \beta} y^{-\frac{\alpha}{1-\alpha}},
$$

and summing up yields the claim.

Lemma 19 We have the inequality

$$
\eta^{(\alpha)}(x) \geq\left(\frac{1-\alpha}{\alpha \beta}\right)^{\frac{1-\alpha}{\alpha}} x^{-\frac{1-\alpha}{\alpha}}, \quad\left(x \geq \frac{\mu^{2}+2(1-\alpha) \beta \sigma^{2}}{2^{\alpha} \mu^{\alpha} \alpha \beta\left(\mu^{2}+2 \beta \sigma^{2}\right)^{1-\alpha}}\right) .
$$


Proof: This follows by applying the previous lemma to $y=\eta(x)$.

We note in passing, that

$$
\lim _{\alpha \rightarrow 1}\left(\frac{\mu^{2}+2(1-\alpha) \beta \sigma^{2}}{2^{\alpha} \mu^{\alpha} \alpha \beta\left(\mu^{2}+2 \beta \sigma^{2}\right)^{1-\alpha}}\right)=\frac{\mu}{2 \beta} .
$$

Lemma 20 We have

$$
\lim _{\alpha \rightarrow 1} \eta^{(\alpha)}(x)=1, \quad(x \geq 0),
$$

and

$$
\lim _{\alpha \rightarrow 1} \frac{1-\alpha}{\alpha}\left(\eta^{(\alpha)}(x)\right)^{-\frac{1}{1-\alpha}}=(\beta x-\mu)_{+} \quad(x \geq 0) .
$$

Proof: (i) A direct calculation shows

$$
\eta^{(\alpha)}(0)=\left(\frac{\alpha}{2(1-\alpha)}\right)^{-(1-\alpha)}\left(\sqrt{\mu^{2}+2 \beta \sigma^{2}(1-\alpha)}-\mu\right)^{-(1-\alpha)}
$$

and

$$
\lim _{\alpha \rightarrow 1} \eta^{(\alpha)}(0)=1
$$

We fix an arbitrary $b>x$, that is sufficiently large to be able to apply Lemma 19. We let $\alpha \rightarrow 1$ and obtain

$$
\liminf _{\alpha \rightarrow 1} \eta^{(\alpha)}(b)=1 \text {. }
$$

As $\eta^{(\alpha)}$ are decreasing, we deduce

$$
\lim _{\alpha \rightarrow 1} \eta^{(\alpha)}(x)=1
$$

uniformly for $0 \leq x \leq b$.

(ii) Next we observe, that for $x>\mu / \beta$ and $\alpha$ sufficiently close to 1 , we have $\eta^{(\alpha)}(x)<\theta^{(\alpha)}(x)$ and thus

$$
0 \leq\left(\eta^{(\alpha)}(x)\right)^{\frac{1}{1-\alpha}} \leq\left(\theta^{(\alpha)}(x)\right)^{\frac{1}{1-\alpha}} .
$$

As a consequence of Lemma 17 the right hand side vanishes as $\alpha \rightarrow 1$ and

$$
\lim _{\alpha \rightarrow 1}\left(\eta^{(\alpha)}(x)\right)^{\frac{1}{1-\alpha}}=0 .
$$


The function $\eta^{(\alpha)}$ satisfies by definition, see Lemma 7 and 9 , the equation

$$
x^{2}+p\left(\eta^{(\alpha)}(x)\right) x+q\left(\eta^{(\alpha)}(x)\right)=0 .
$$

We multiply this equation with $\beta^{2}\left(\eta^{(\alpha)}(x)\right)^{\frac{1}{1-\alpha}}$ and deduce

$$
\lim _{\alpha \rightarrow 1} \frac{1-\alpha}{\alpha}\left(\eta^{(\alpha)}(x)\right)^{-\frac{1}{1-\alpha}}=\beta x-\mu .
$$

For $0 \leq x \leq \mu / \beta$ we invoke monotonicity.

Lemma 21 We have

$$
\lim _{\alpha \rightarrow 1} y^{(\alpha)}(x)=1, \quad\left(x>\frac{\mu}{\beta}\right),
$$

and

$$
\lim _{\alpha \rightarrow 1} \frac{1-\alpha}{\alpha}\left(y^{(\alpha)}(x)\right)^{-\frac{1}{1-\alpha}}=(\beta x-\mu)_{+}, \quad(x \geq 0) .
$$

The second limit is uniform on compact subsets of $[0,+\infty)$.

Proof: Lemma 20, Lemma 17, and the inequality

$$
\eta^{(\alpha)}(x) \leq y^{(\alpha)}(x) \leq \theta^{(\alpha)}(x)
$$

imply

$$
\lim _{\alpha \rightarrow 1} \frac{1-\alpha}{\alpha}\left(y^{(\alpha)}(x)\right)^{-\frac{1}{1-\alpha}}=\beta x-\mu, \quad\left(x \geq \frac{\mu}{\beta}\right) .
$$

For $0 \leq x \leq \mu / \beta$ we choose $\epsilon>0$ and invoke the monotonicity of $y^{(\alpha)}$

$$
y^{(\alpha)}\left(\frac{\mu+\epsilon}{\beta}\right) \leq y^{(\alpha)}(x)
$$

thus

$$
0 \leq \frac{1-\alpha}{\alpha}\left(y^{(\alpha)}(x)\right)^{-\frac{1}{1-\alpha}} \leq \frac{1-\alpha}{\alpha}\left(y^{(\alpha)}\left(\frac{\mu+\epsilon}{\beta}\right)\right)^{-\frac{1}{1-\alpha}} .
$$


The right hand side tends by (B.41) above to $\epsilon$. As $\epsilon>0$ was arbitrary, we have

$$
\lim _{\alpha \rightarrow 1} \frac{1-\alpha}{\alpha}\left(y^{(\alpha)}(x)\right)^{-\frac{1}{1-\alpha}}=0, \quad\left(0 \leq x \leq \frac{\mu}{\beta}\right) .
$$

Now fix an arbitrary $b>\mu / \beta$. In (B.41) we have a sequence of increasing functions that tends to a continuous limit on the compact interval $[0, b]$. Thus we must have uniform convergence on such intervals.

\section{B.3 Bounds and limits for the ODE solution}

Lemma 22 We have

$$
\liminf _{\alpha \rightarrow 1} y^{(\alpha)}(x) \geq 1, \quad(x \in \mathbb{R}),
$$

and

$$
\begin{aligned}
& \limsup _{\alpha \rightarrow 1} y^{(\alpha)}(x) \leq 1+\frac{4 \mu^{2}}{\beta \sigma^{2}}, \quad(x \geq 0), \\
& \limsup _{\alpha \rightarrow 1} y^{(\alpha)}(x) \leq 1+\frac{4 \mu^{2}}{\beta \sigma^{2}}+\frac{\beta x^{2}-2 \mu x}{\sigma^{2}}, \quad(x \leq 0) .
\end{aligned}
$$

Proof: Equation (B.45) follows from $y(x) \geq \eta(x)$ and (B.28) in Lemma 20. As $y$ is a decreasing function, it is sufficient to show (B.46) for $x=0$ : We use $y(x) \geq \eta\left(\hat{x}_{1}\right) \geq \ell\left(\hat{x}_{1}\right)$ for $0 \leq x \leq \hat{x}_{1}$, where $\ell(x)=\gamma_{\alpha} x^{-\frac{1-\alpha}{\alpha}}$. This implies

$$
F(z, y(z)) \geq \frac{2}{\sigma^{2}}\left(-\frac{1-\alpha}{\alpha} \ell\left(\hat{x}_{1}\right)^{-\frac{1}{1-\alpha}}-\mu\right)=F\left(0, \ell\left(\hat{x}_{1}\right)\right), \quad\left(0 \leq z \leq \hat{x}_{1}\right) .
$$

Thus

$$
\begin{aligned}
y(0) & =y\left(\hat{x}_{1}\right)-\int_{0}^{\hat{x}_{1}} F(z, y(z)) d z \\
& \leq \theta\left(\hat{x}_{1}\right)+\left(\frac{1-\alpha}{\alpha} \ell\left(\hat{x}_{1}\right)^{-\frac{1}{1-\alpha}}+\mu\right) \hat{x}_{1}=: b^{(\alpha)}(0) .
\end{aligned}
$$

Next we consider $x \leq 0$ : We use $y(x) \geq \eta(0)$ for $x \leq 0$. This implies 


$$
\begin{aligned}
F(z, y(z)) & \geq \frac{2}{\sigma^{2}}\left(\frac{\beta z}{\eta(0)}-\frac{1-\alpha}{\alpha} \eta(0)^{-\frac{1}{1-\alpha}}-\mu\right) \\
& =F(z, \eta(0)), \quad(x \leq z \leq 0)
\end{aligned}
$$

Thus

$$
\begin{aligned}
y(x) & =y(0)-\int_{x}^{0} F(z, y(z)) d z \\
& \leq b^{(\alpha)}(0)-\frac{2}{\sigma^{2}} \int_{x}^{0}\left(\frac{\beta z}{\eta(0)}-\frac{1-\alpha}{\alpha} \eta(0)^{-\frac{1}{1-\alpha}}-\mu\right) d z \\
& =b^{(\alpha)}(0)+\frac{2}{\sigma^{2}}\left(\frac{\beta x^{2}}{2 \eta(0)}-\left(\frac{1-\alpha}{\alpha} \eta(0)^{-\frac{1}{1-\alpha}}+\mu\right) x\right)=: b^{(\alpha)}(x)
\end{aligned}
$$

Finally a direct calculation shows

$$
\lim _{\alpha \rightarrow 1} b^{(\alpha)}(x)=1+\frac{4 \mu^{2}}{\beta \sigma^{2}}+\frac{\beta x^{2}-2 \mu x}{\sigma^{2}}, \quad(x \in \mathbb{R}) .
$$

\section{References}

Albrecher, H., Kainhofer, R., 2002. Risk theory with a non-linear dividend barrier. Computing 68 (4), 289-311.

Albrecher, H., Kainhofer, R., Tichy, R., 2003. Simulation methods in ruin models with non-linear dividend barriers. Math. Comput. Simulation 62 (36), 277-287.

Asmussen, S., Taksar, M., 1997. Controlled diffusion models for optimal dividend pay-out. Insurance Math. Econom. 20 (1), 1-15.

Birkhoff, G., Rota, G.-C., 1969. Ordinary differential equations. Second edition. Blaisdell Publishing Co. Ginn and Co., Waltham, Mass.-Toronto, Ont.London.

Björk, T., Myhrman, J., Persson, M., 1987. Optimal consumption with stochastic prices in continuous time. J. Appl. Probab. 24 (1), 35-47.

Boogaert, P., Delbaen, F., Haezendonck, J., 1988. Macro-economic influences on the crossing of dividend barriers. Scand. Actuar. J. (4), 231-245.

Conte, L., 1932. Sull'integrazione dell'equatione differenziale $y^{\prime}+p y^{r}+q y^{s}=0$. Boll. Unione Math. Ital. 32, 216-219.

Corless, R. M., Gonnet, G. H., Hare, D. E. G., Jeffrey, D. J., Knuth, D. E., 1996. On the Lambert $W$ function. Adv. Comput. Math. 5 (4), 329-359.

Feller, W., 1971. An introduction to probability theory and its applications. Vol. II., 2nd Edition. John Wiley \& Sons Inc., New York. 
Gerber, H., 1974. The dilemma between dividends and safety and a generalization of the Lundberg-Cramér formulas. Scand. Actuar. J., 46-57.

Gerber, H. U., 1979. An introduction to mathematical risk theory. Vol. 8 of S.S. Heubner Foundation Monograph Series. University of Pennsylvania Wharton School S.S. Huebner Foundation for Insurance Education, Philadelphia, $\mathrm{Pa}$.

Gerber, H. U., 1981. On the probability of ruin in the presence of a linear dividend barrier. Scand. Actuar. J. (2), 105-115.

Gjessing, H. K., Paulsen, J., 1997. Present value distributions with applications to ruin theory and stochastic equations. Stochastic Processes Appl. 71 (1), 123-144.

Helly, E., 1912. Über lineare Funktionaloperationen. Sitzungsberichte der Kaiserlichen Akademie der Wissenschaften, MathematischNaturwissenschaftliche Klasse 121, 265-297.

Højgaard, B., Taksar, M., 1999. Controlling risk exposure and dividends payout schemes: Insurance company example. Math. Finance 9 (2), 153-182.

Kamke, E., 1977. Differentialgleichungen. B. G. Teubner, Stuttgart.

Karatzas, I., Lehoczky, J. P., Shreve, S. E., Xu, G.-L., 1991. Martingale and duality methods for utility maximization in an incomplete market. SIAM J. Control Optim. 29 (3), 702-730.

Kramkov, D., Schachermayer, W., 1999. The asymptotic elasticity of utility functions and optimal investment in incomplete markets. Ann. Appl. Probab. 9 (3), 904-950.

Paulsen, J., 1998. Ruin theory with compounding assets - a survey. Insur. Math. Econ. 22 (1), 3-16.

Pólya, G., Szegö, G., 1925. Aufgaben und Lehrsätze aus der Analysis. Band I: Reihen, Integralrechnung, Funktionentheorie. Springer-Verlag, Berlin.

Rockafellar, R. T., 1970. Convex analysis. Princeton Mathematical Series, No. 28. Princeton University Press, Princeton, N.J.

Rudin, W., 1987. Real and complex analysis, 3rd Edition. McGraw-Hill Book Co., New York.

Siegl, T., Tichy, R. F., 1999. A process with stochastic claim frequency and a linear dividend barrier. Insur. Math. Econ. 24 (1-2), 51-65.

Siegl, T., Tichy, R. F., 2000. Ruin theory with risk proportional to the free reserve and securitization. Insur. Math. Econ. 26 (1), 59-73.

Taksar, M. I., 2000. Optimal risk and dividend distribution control models for an insurance company. Math. Methods Oper. Res. 51 (1), 1-42.

Taksar, M. I., Zhou, X. Y., 1998. Optimal risk and dividend control for a company with a debt liability. Insur. Math. Econ. 22 (1), 105-122.

Waldmann, K.-H., 1988. On optimal dividend payments and related problems. Insurance Math. Econom. 7 (4), 237-249. 